\title{
L'industrie lithique de la couche III du Roc de Marsal (Dordogne) : le problème de l'attribution d'une série lithique au Moustérien à denticulés
}

The industry of layer III from the site of Roc de Marsal: The problem of its attribution to the Denticulate Mousterian

\section{Céline Thiebaut}

\section{OpenEdition \\ Journals}

Édition électronique

URL : http://journals.openedition.org/paleo/1274

DOI : $10.4000 /$ paleo. 1274

ISSN : 2101-0420

Éditeur

SAMRA

Édition imprimée

Date de publication : 1 décembre 2003

Pagination : 141-168

ISSN : 1145-3370

Référence électronique

Céline Thiebaut, «L'industrie lithique de la couche III du Roc de Marsal (Dordogne) : le problème de l'attribution d'une série lithique au Moustérien à denticulés », PALEO [En ligne], 15 | 2003, mis en ligne le 03 août 2010, consulté le 07 juillet 2020. URL : http://journals.openedition.org/paleo/1274 ; DOI : https://doi.org/10.4000/paleo.1274

Ce document a été généré automatiquement le 7 juillet 2020

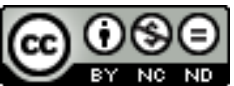

PALEO est mis à disposition selon les termes de la licence Creative Commons Attribution - Pas d'Utilisation Commerciale - Pas de Modification 4.0 International. 


\section{L'industrie lithique de la couche III du Roc de Marsal (Dordogne) : le problème de l'attribution d'une série lithique au Moustérien à denticulés}

The industry of layer III from the site of Roc de Marsal: The problem of its attribution to the Denticulate Mousterian

\section{Céline Thiebaut}

\section{Introduction}

1 L'industrie moustérienne a été définie à la fin du XIXème siècle comme un ensemble dominé par les outils sur éclats tels que les racloirs et les pointes (Mortillet 1872). Assez rapidement, s'est imposé la nécessité de distinguer des sous-groupes au sein de cet ensemble en fonction de la présence d'outils autres que ceux du fonds commun. Les travaux précurseurs de D. Peyrony ont par exemple conduit à la définition du Moustérien de Tradition Acheuléenne sur la base de la présence de couteaux à dos et de bifaces (Peyrony 1930). À partir des années 1950, sous l'impulsion de F. Bordes et M. Bourgon, cette démarche s'est systématisée en même temps qu'elle a gagné en précision.

2 L'utilisation d'outils statistiques simples permet notamment de prendre en considération, non pas la présence ou l'absence de quelques fossiles directeurs, mais les fréquences relatives d'outils ou de groupes d'outils sur la totalité de l'outillage des séries étudiées. L'analyse typologique de plusieurs séries archéologiques de référence a 
ainsi conduit $\mathrm{F}$. Bordes à définir - ou redéfinir - cinq groupes principaux au sein du Moustérien d'Europe occidentale :

- le Moustérien de type Quina ;

- le Moustérien de type Ferrassie ;

- le Moustérien typique ;

- le Moustérien de Tradition Acheuléenne ;

- et enfin, le Moustérien à denticulés (Bordes 1953).

3 Ces groupes sont qualifiés de "faciès" par analogie avec les faciès géologiques et sont considérés par $\mathrm{F}$. Bordes comme le reflet de cinq traditions culturelles distinctes. L'interprétation de ces faciès a fait l'objet de vives polémiques et plusieurs autres hypothèses ont été proposées :

- hypothèse économique (Binford 1966) ;

- hypothèse chronologique (Mellars 1969);

- diversité liée à l'intensité des cycles de ravivage pour les faciès charentiens (Dibble 1984) ;

- déterminisme des matières premières (Geneste 1985) ;

- influence du contexte topographique et géographique (Barton 1989);

- hypothèse environnementale (Rolland 1990).

4 Toutefois, aucune d'entre elles ne semble s'imposer unanimement.

5 Le Moustérien à denticulés est donc l'un des faciès définis par F. Bordes. Sa définition repose sur l'analyse typologique de séries lithiques issues de 28 couches archéologiques de 14 gisements dont un grand nombre dans le Sud-Ouest de la France. Toutes ces séries présentent un fort pourcentage de denticulés et d'encoches.

6 Selon F. Bordes, les caractéristiques de ce faciès sont les suivantes :

“Industrie pauvre en racloirs ${ }^{1}$ très pauvre en pointes, peu ou pas de bifaces toujours atypiques, le plus souvent nucléiformes ou partiels.

Peu ou pas de couteaux à dos, le plus souvent atypiques. Présence d'un très fort pourcentage d'encoches (9 à $46 \%$ ) et de denticulés (20 à $48 \%$ ). Facture variable, parfois bonne, souvent médiocre.

Débitage Levallois ou non. Pourcentage variable de talons facettés. Il y a toujours plus d'outils denticulés que de racloirs" (Bordes 1962-63, p. 44).

7 En 1984, F. Bordes précise que l'on ne trouve “ jamais de racloir Quina " dans le Moustérien à denticulés. La rareté des racloirs, caractéristique essentielle de ce faciès avait déjà été énoncée par M. Bourgon dans son ouvrage "Les industries moustériennes et pré-moustériennes du Périgord " (Bourgon 1957). En effet, selon lui, plus que le fort pourcentage de denticulés que l'on peut retrouver dans d'autres industries, "la carence des autres formes bien définies, caractéristiques habituellement du Moustérien, laisse la prédominance aux éclats denticulés " (Bourgon 1957, p. 109).

8 Ce faciès paraît alors très particulier puisqu'il se caractérise, paradoxalement, par l'absence de certains types d'outils et par la faible proportion de racloirs, généralement "très médiocres » (Bordes 1981, p. 79), qui sont pourtant caractéristiques du Moustérien en général.

9 Au sein de ce faciès, quatre " groupes technologiques " ont été distingués en fonction du pourcentage d'éclats Levallois bruts et retouchés et du facettage des talons des éclats :

- le Moustérien à denticulés à débitage Levallois et faciès levalloisien : il présente un indice d'éclats Levallois bruts ou retouchés (IL) supérieur à $20 \%$ sur l'ensemble des éclats et un fort 
indice d'éclats Levallois non retouchés ( $\mathrm{IL}^{\text {ty }}>$ à $35 \%$ ). F. Bordes y rattache les industries de la carrière d'Evreux II et les couches 11 et 38 de Combe-Grenal ;

- le Moustérien à denticulés à débitage Levallois et faciès non levalloisien : la seule différence avec le groupe précédent est le fait que son indice d'éclats Levallois bruts (IL ${ }^{\text {ty }}$ ) est inférieur à $35 \%$. Ce serait le cas pour les industries de la couche VI de l'Abri Suard et pour le gisement de la Métairie ;

- le Moustérien à débitage non-Levallois et facetté : il présente un faible indice d'éclat Levallois (IL $<20 \%)$ mais un indice de facettage (IF) des talons de tous les éclats supérieurs à $45 \%$. F. Bordes cite pour exemple l'industrie de la couche 4 du Pech de l'Azé II ;

- le Moustérien à débitage non-Levallois et non facetté : outre un indice d'éclats Levallois inférieur à $20 \%$, l'indice de facettage est inférieur à $45 \%$. Ces caractéristiques ont été observées par F. Bordes au sein de l'industrie de la couche 14 de Combe-Grenal (Bordes 1953 et 1962-63).

Il en ressort que ce faciès se caractérise typologiquement par un grand nombre d'encoches et de denticulés et qu'il ne présente pas de caractéristique technologique spécifique : le débitage pouvant être Levallois ou non.

11 Les années 1980 ont vu le développement des analyses technologiques (Geneste 1985) qui intègrent l'outillage dans une chaîne opératoire plus complexe, depuis l'acquisition des matières premières jusqu'à l'abandon des pièces lithiques. Mais, même si de nombreux travaux montrent l'importance d'une analyse technologique globale des industries pour une meilleure caractérisation des faciès moustériens, l'attribution d'une industrie à un faciès Moustérien dépend toujours du type d'outil qui prédomine. Cette attribution découle nécessairement de la reconnaissance typologique des outils retouchés. Cependant, il existe un obstacle constant à l'identification de certains faciès du Moustérien: la reconnaissance typologique de certaines pièces comme étant de véritables outils. En effet, la présence de pseudo-outils au sein de séries lithiques préhistoriques est connue de longue date dans plusieurs gisements (Bordes et Bourgon 1951b ; Bordes 1961). La difficulté réside alors dans la reconnaissance formelle de ces pièces. Cette reconnaissance est encore plus difficile lorsqu'il s'agit de denticulés ou d'encoches. Cela paraît alors paradoxal lorsque l'on sait que ces objets sont caractéristiques de l'un des faciès du Paléolithique moyen : le Moustérien à denticulés.

La présence de pseudo-outils encochés au sein des séries lithiques préhistoriques, a été évoquée dès la fin des années 30 (Pei 1936). Depuis, de nombreux auteurs soulignent dans leurs travaux la difficulté qu'il y a parfois à différencier les outils retouchés et, $a$ fortiori les pièces encochées des " pseudo-outils" et des outils a posteriori (Bordes 1970 ; Girard 1978; Chavaillon 1985; Verjux 1988; Bernard-Guelle 1995). De nombreux travaux ont alors été orientés vers la caractérisation de ce type de pièces. Ainsi, à partir des années 1950 plusieurs chercheurs ont tenté, à la fois par l'observation du matériel archéologique et par la mise en place de programmes expérimentaux, de caractériser les pseudo-outils afin de permettre leur identification au sein de séries lithiques. Sans avoir la prétention d'être exhaustive, nous pouvons citer les travaux de F. Bordes et M. Bourgon ainsi que ceux de F. Pradel, de J. Tixier et R. Mallouf concernant les caractéristiques des négatifs des enlèvements des pseudo-outils (Bordes et Bourgon 1951b; Pradel 1956; Bordes 1961; Tixier 1963; Mallouf 1982). Néanmoins, ces caractéristiques représentent une tendance générale et ne sont pas systématiquement synonymes d'enlèvements accidentels ou naturels (Bordes et Bourgon, 1951a, Bordes, 1962-1963). Plusieurs expériences et programmes expérimentaux ont aussi été 
orientées vers la production de bords encochés lors de piétinement humain ou animal (Tringham et al. 1974 ; Knudson 1979 ; Flennikenn et Haggarty 1979 ; Prost1989 ; Nielsen 1991 ; Mc Brearty et al. 1998), lors de l'utilisation d'un tranchant brut (Beyries 1987; Prost op.cit.) lors du débitage (Newcomer 1976; Ploux 1983; Mansur-Franchomme 1986 ; Prost op.cit. ; Inizan et al. 1995) ou encore lors de la confection d'une retouche sur un tranchant brut (Fournier 1973 ; Pros, op. cit. ; Benito del Rey et Benito Alvarez 1998)2

Tous ces phénomènes jouent donc un rôle prépondérant dans l'interprétation d'une industrie lithique. En effet, si les pseudo-outils sont nombreux au sein d'une industrie lithique, ils sont susceptibles d'entraîner des erreurs lors de l'attribution d'un ensemble à un faciès du Paléolithique moyen, notamment le Tayacien (Bordes 1970) et le Moustérien à denticulés (Bordes 1961,1962-1963, 1984).

Dans cette optique, nous avons donc tenté de distinguer les pièces encochées des pseudo-outils au sein de l'industrie de la couche III du Roc de Marsal. Pour ce faire, nous avons utilisé une méthode d'étude proposée dans le cadre d'un travail universitaire (Thiébaut 2001) qui synthétise notamment les observations et les travaux effectués par d'autres chercheurs.

\section{Présentation générale du site}

Le Roc de Marsal est un abri-sous-roche localisé au cœur du Périgord noir, dans un contexte très dense en occupations préhistoriques du Paléolithique (fig. 1).

Figure 1 - Localisation du Roc de Marsal en Dordogne et des principaux sites paléolithiques du Périgord.

Figure 1 - Location of the Roc de Marsal site in Dordogne and the Paleolithic sites of Périgord.

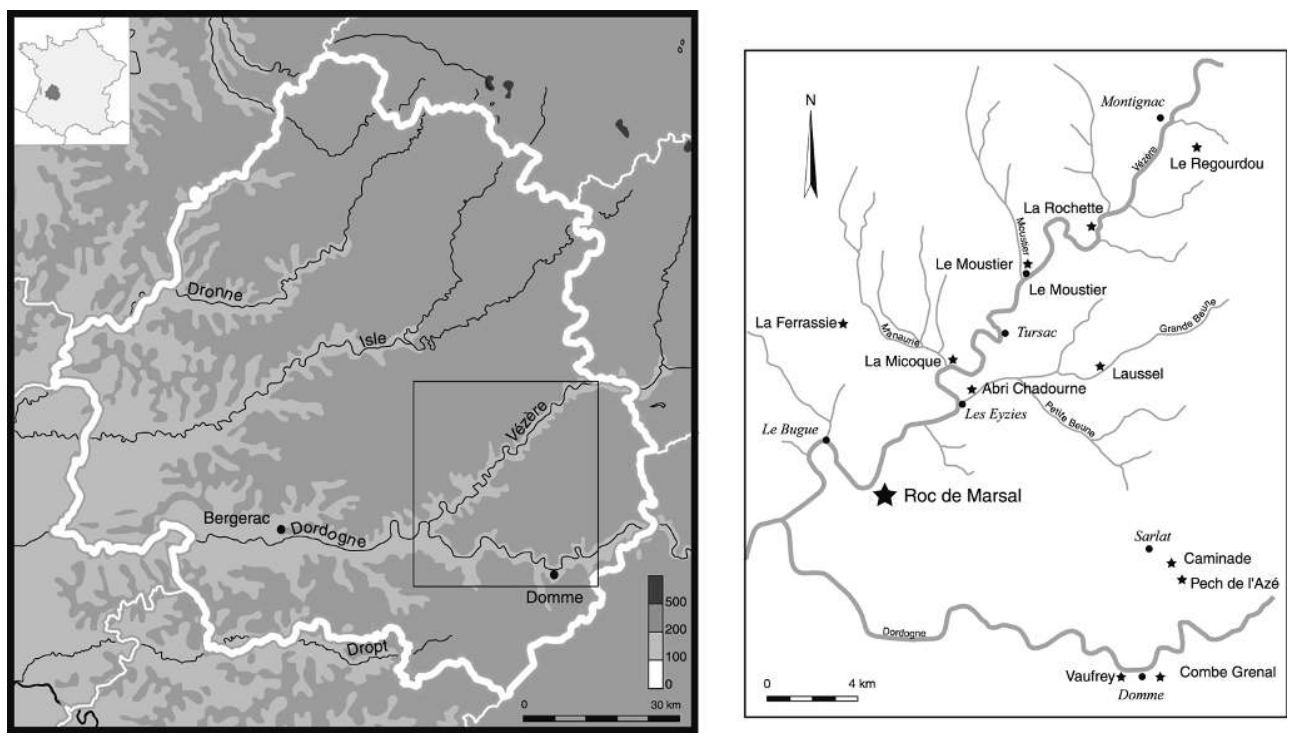

Il se situe dans le vallon de la Redonde, sur la commune de Campagne (Dordogne). Le relief y est marqué par la présence de l'anticlinal de Saint-Cyprien (Séronie-Vivien 1959 ; Konik 1999) à l'origine de la formation d'une ligne de falaises. Le réseau karstique présente trois cavités (cf. fig. I. Couchoud même ouvrage) :

- une grotte d'ampleur importante obstruée par des blocs d'effondrement ;

- une cavité de plus petite taille qui, selon J. Bouchereau (1967), devait être reliée à la première ; 
- le Roc de Marsal proprement dit, un abri de 5,50 mètres de largeur sur 9 mètres de profondeur et d'une hauteur de près de 3 mètres qui se situe à $140 \mathrm{~m}$ au-dessus du lit de la Vézère. Son plafond est parcouru par une faille orientée sud-ouest/nord-est et dont l'élargissement par dissolution est à l'origine de la formation de l'abri (Assassi 1986). 1961). L'inventeur entreprit un sondage l'année suivante qui révéla la présence d'industries moustériennes en place. Le site fut immédiatement carroyé avant le début des fouilles qui commencèrent au cours de l'été 1954. De 1954 à 1971, date de sa mort, J. Lafille fouilla seul les quatorze couches sur une surface de $27 \mathrm{~m}^{2}$. Sa méthode consista à fouiller les carrés les uns après les autres. Il cartographia un quart du matériel relevé, prit de nombreuses notes sur ses cahiers de fouille, déposés au Musée National de Préhistoire des Eyzies, et releva 30 mètres de coupes stratigraphiques. C'est pendant la campagne de fouille de 1954 et celle de 1955 qu'il put, à partir de la fouille du carré L17, séparer 14 unités stratigraphiques numérotées de I à XIV, de bas en haut. Plus tard, la fouille des carrés $M 17, \mathrm{~L} 16, \mathrm{~L} 18$, montra que la couche I n'était pas la roche mère et ainsi, trois autres couches furent distinguées et nommées: A, B, C, de haut en bas (Lafille n. d. ; Turq 1989).

campagne de fouille de l'année 1961 permit la mise au jour du squelette d'un enfant néandertalien dont l'âge est estimé à trois ans (Bordes et Lafille 1962; Legoux 1965; Madre-Dupouy 1988). Le caractère anthropique de la fosse dans laquelle l'enfant a été retrouvé a été discuté par la suite (Defleur 1987 et 1993 ; Turq 1989).

\section{Stratigraphie et mise en place des dépôts}

La stratigraphie du Roc de Marsal, décrite par J. Lafille, puis par les travaux de J. Bouchud (1975), F. Assasi (1986) et A. Turq (1979, 1988, 1989, fig. 2A) a été récemment réétudiée par I. Couchoud (Couchoud 2001 et à paraître). Les données issues de ces travaux concernant l'analyse sédimentologique et la mise en place des dépôts sont synthétisées ci-dessous.

Si l'on se réfère à la coupe A (fig. 2B), 5 unités stratigraphiques ont été distinguées au sein du remplissage (tabl. 1). 
Figure 2 - Roc de Marsal. A : coupe stratigraphique (Turq, 1979), B : coupe stratigraphique A (Couchoud, 2003)

Figure 2 - Roc de Marsal. A Stratigraphic section (Turq 1979), B, Stratigraphic section A (Couchoud 2003)

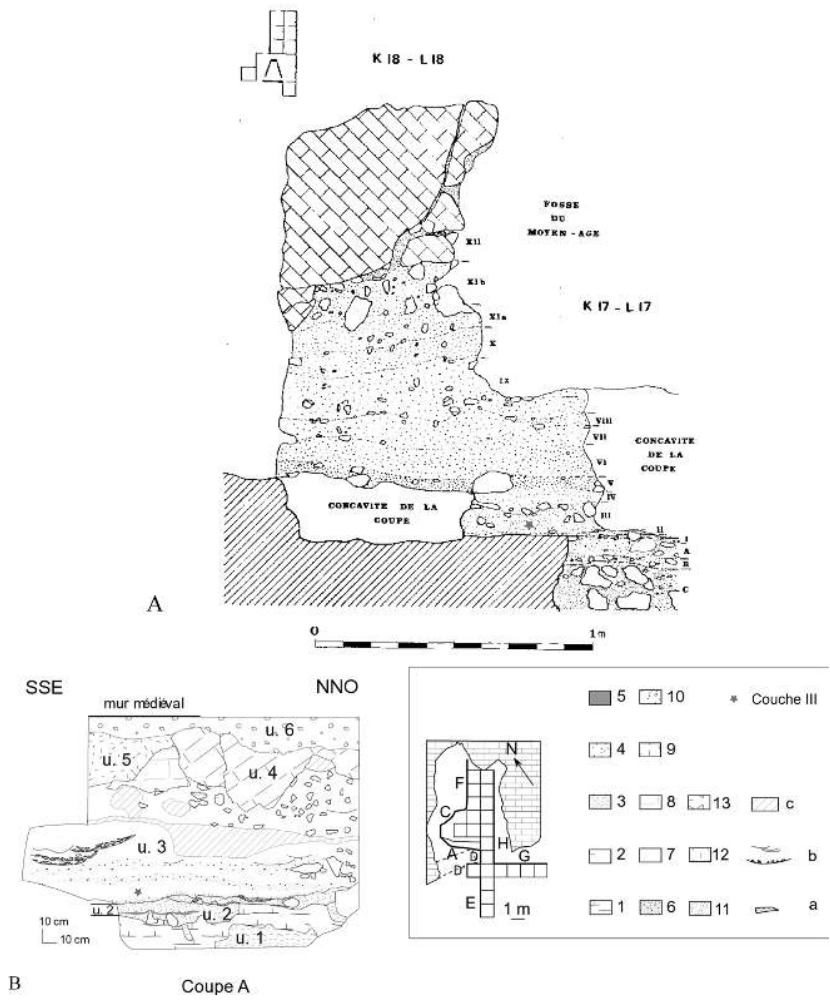

Légende : 1 : substratum. 2 : substratum désagrégé. 3 : sables triés dans une matrice d'argile rouge 4 : sables grossiers quartzeux et calcaires. 5 : niveau rubéfié. 6 : passées charbonneuses. $7:$ sables limoneux à argileux, plus ou moins bioturbés et riches en vestiges d'origine anthropique. 8 : blocs calcaires effondrés. 9 : diamicton à matrice sablo-argileuse, localement carbonaté. 10 : dépôts superficiels remaniés. 11 : cendres. 12 : horizon carbonate. 13 : sol organique. a : matériel archéologique. b : lentille grossière ou sableuse. c : décrochement du plan de coupe et perturbations importantes (terriers, silo).

Legend: 7: bedrock. 2: disaggregated bedrock. 3: sorted sands in a red clayey matrix. 4: quartzous and calcareous coarse sands. 5: reddish level. 6: charcoal-rich levels. 7: loamy to clayey sands, more or less bioturbated and rich in anthropogenic remains. 8: limestone slab break down. 9: diamicton with a silty-clayey matrix, locally carbonated. 10: surface reworked deposits. 11: ashes. 12: carbonated horizon. 13: organic soil. a: archaeological material. b: coarse or sandy lens. c: offsetting in the section plan and important perturbations (burrow, silo). 
Tableau 1 - Présentation synthétique des données sédimentologiques (coupe $A$ ) et des processus de mise en place des dépôts (d'après les données de I. Couchoud 2001 et dans ce volume).

Table 1 - Synthetic presentation of sedimentological data (section A) and of site formation processes (adapted from I. Couchoud 2001 and in this issue).

\begin{tabular}{|c|c|c|c|}
\hline Turq 1979 & $\begin{array}{c}\text { Couchoud } \\
2003\end{array}$ & Description & $\begin{array}{l}\text { Processus de mise en place des } \\
\text { dépôts }\end{array}$ \\
\hline c XIV & Unité 5 & $\begin{array}{l}\text { Épaisseur moyenne de } 25 \mathrm{~cm} \text {. Repose sur les blocs } \\
\text { effondrés et sur l'unité } 4 \text { avec une limite floue. Dépôts } \\
\text { superficiels, avec épandage cendreux. Hétérogène, } \\
\text { composée d'un sable limoneux fortement bioturbé et mal } \\
\text { trié. Couleur brun jaunâtre. } \\
\text { Épaisseur max. } 35 \mathrm{~cm} \text {. Sol organique vers le haut et } \\
\text { carbonatation vers le bas. Sables limoneux massifs triés } \\
\text { essentiellement quartzeux et carbonatés, plus quelques } \\
\text { graviers et cailloutis calcaire. Couleur jaune. } \\
\text { De } 20 \text { à } 50 \mathrm{~cm} \text { d'épaisseur, blocs d'effondrement en grande } \\
\text { partie pris dans les dépôts de l'unité } 2 \text {. }\end{array}$ & $\begin{array}{l}\text { éboulisation, remaniement et } \\
\text { sédimentation d'origine } \\
\text { anthropique }\end{array}$ \\
\hline $\begin{array}{l}\mathrm{c} \mathrm{XI} \\
\text { à } \\
\mathrm{c} \mathrm{II}\end{array}$ & Unité 3 & $\begin{array}{l}\text { Épaisseur max. } 90 \mathrm{~cm} \text {. Limite inférieure nette. Dépôts à } \\
\text { matrice sablo-limoneuse et à fraction grossière abondante. } \\
\text { La bioturbation est très importante et structure globalement } \\
\text { la matrice en agrégats. De manière localisée, une structure } \\
\text { lamellaire se devine. }\end{array}$ & $\begin{array}{l}\text { ruissellement, sédimentation } \\
\text { anthropique, éboulisation } \\
\text { variable. }\end{array}$ \\
\hline $\begin{array}{l}\mathrm{c} \mathrm{I} \\
\mathrm{c} \mathrm{A} \\
\mathrm{c} \mathrm{B} \\
\mathrm{c} \mathrm{C}\end{array}$ & Unité 2 & $\begin{array}{l}\text { De } 3 \text { à } 20 \mathrm{~cm} \text {, repose sur le substratum. Limite inférieure } \\
\text { érosive. Dépôt à matrice sablo-limoneuse pauvre en } \\
\text { matériel archéologique et faiblement bioturbé. }\end{array}$ & ruissellement \\
\hline & Substratum & $\begin{array}{l}\text { Remplissage de poches karstiques développées au plancher } \\
\text { de la cavité. Sables quartzeux massifs, parfois limoneux. } \\
\text { Intercalations de taches et de niveaux horizontaux très } \\
\text { argileux. De couleur rouge jaunâtre ou brun vif avec des } \\
\text { taches brun jaunâtre. } \\
\text { Calcaire de type grainstone, riche en grains quartzeux } \\
\text { grossiers bien triés, bioclastique, peletoïdal, de couleur } \\
\text { jaune. Il se résout en fragments aplatis de plus en plus } \\
\text { minces vers le haut avant de devenir complètement } \\
\text { meuble. Présente une structure lamellaire dans la partie } \\
\text { meuble. }\end{array}$ & $\begin{array}{l}\text { ruissellement, remplissage de } \\
\text { poches karstiques }\end{array}$ \\
\hline
\end{tabular}

Il est possible de repositionner la couche III individualisée par le fouilleur sur la coupe relevée par I. Couchoud. En effet, à l'issue d'une étude comparative des deux représentations stratigraphiques (Couchoud 2001; Turq 1979) et des données sédimentologiques provenant des travaux de I. Couchoud et F. Assassi, la couche III décrite comme un niveau brun foncé (H 64) comprise entre deux couches plus grises (Turq op.cit.), pourrait correspondre à la couche claire reposant sur les niveaux charbonneux basaux de l'unité 3 et présentée sur la coupe $\mathrm{A}^{3}$ (fig. 2B).

\section{Attribution culturelle et données environnementales}

Fouillée dans les années 60, la majorité des couches archéologiques identifiées a été attribuée à des occupations préhistoriques moustériennes.

En ce qui concerne les études paléontologiques et environnementales effectuées, nous ne pouvons que déplorer le fait que la faune n'ait fait l'objet que d'une étude préliminaire (Bouchud 1975) et que l'étude des pollens n'ait été réalisée que dans la couche I (Van Campo et al. 1962). Toutefois, l'étude de la faune a permis de mettre en évidence certaines tendances générales. Les couches I et II ont livré quelques vestiges de Cerf élaphe, Alectoris barbara et Capreolus, qui indiquent un climat peu rigoureux. La couche III est marquée par une dégradation climatique avec l'apparition du Renne associé aux Bovidés, équidés et Cervidés. Dès la couche IV, le Renne devient l'animal dominant et la proportion des chevaux augmente jusqu'à la couche X. Le Renard et le Lièvre font leur apparition dès la couche VII. 
À partir de l'étude préliminaire de l'industrie lithique, trois faciès ont été reconnus : un Moustérien de type Quina, un Moustérien typique et un Moustérien à denticulés (Bordes cité par Turq 1979). Cette première attribution a été établie à partir d'un échantillon du matériel.

L'attribution des séries provenant des couches VIII, IX, X, XI et XII à un faciès moustérien de type Quina a été confirmée par les travaux de A. Turq (Turq 1979, 1989, 1992). Le travail de G. Antignac a confirmé l'attribution de la couche II au Moustérien à denticulés, tandis que l'étude de la couche I a été abandonnée car elle présentait de nombreuses difficultés, liées au faible effectif de vestiges lithiques et au fort pourcentage d'enlèvements non anthropiques (Antignac 1998). Cet auteur s'est donc concentré sur l'étude typo-technologique de la couche II qui a livré près de 5000 pièces lithiques. Les données issues de son étude et confrontées à celles d'autres séries du Moustérien à denticulés ainsi qu'aux caractéristiques de ce faciès définies par F. Bordes, lui permettent d'attribuer l'industrie de cette couche au Moustérien à denticulés de débitage Levallois (Antignac, op.cit.).

L'industrie lithique de la couche III du Roc de Marsal a été attribuée avec certaines réserves au Moustérien à denticulés du fait de la présence soupçonnée de pseudo-outils au sein du groupe IV (Thiébaut 2000, p. 35 et p. 103).

L'objectif de cet article consiste à s'interroger sur les caractères discriminant d'une série lithique lors de son rattachement au Moustérien à denticulés. En effet, un réexamen des pièces encochées, effectué en juin 2002 à partir d'une méthode élaborée dans un travail antérieur (Thiébaut 2001), laisse supposer que la présence de pseudooutils a entraîné une surestimation du groupe IV (encoches et denticulés) lors de notre première étude.

\section{État de conservation des vestiges de la couche III du Roc de Marsal}

Selon l'analyse de I. Couchoud, le ruissellement, associé à de l'éboulisation et à une sédimentation d'origine anthropique, semble être le principal agent de mise en place de la couche III.

D’une manière générale, les phénomènes post-dépositionnels qui ont affecté le matériel de la couche III ont entraîné de nombreuses altérations qui ont eu pour conséquence des transformations physico-chimiques de la matière. La grande majorité des tranchants présente des négatifs d'enlèvements naturels, certaines pièces présentent une double patine et d'autres, de manière plus sporadique, un émoussé total de la pièce.

Nous avons aussi constaté la présence de nombreux indices de chauffe accidentelle ( $\mathrm{n}=$ 790 , soit $19,7 \%$ du matériel) qui pourraient être liés à la présence de plusieurs foyers. En effet, si l'on se réfère aux données issues des cahiers de fouilles, l'existence de foyers contemporains est mentionnée à plusieurs reprises (Lafille, n.d.).

31 Il existe de surcroît un nombre important d'objets fragmentés $(n=826$ pour les éclats bruts seulement), en partie lié à un effondrement d'une partie du plafond de la cavité (présence de gros blocs d'effondrement). 


\section{Analyse économique et technologique de l'industrie de la couche III du Roc de Marsal}

Géologiquement, l'abri du Roc de Marsal est implanté dans des formations calcaires compactes du Turonien directement sous-jacentes au niveau coniacien à silex (A. Morala com. pers.) (fig. 3). Le massif coniacien a été démantelé par l'érosion et parfois recouvert ou repris par des sédiments tertiaires à tendance sidérolithique. Localement, cet environnement sédimentaire a favorisé une altération de certains matériaux siliceux (A. Morala in Thiébaut, 2000) ; ainsi, sur le plateau et sur ses flancs, des blocs de silex à cortex altéré coexistent avec d'autres qui présentent un cortex frais.

Figure 3 - Carte géologique simplifiée et localisation du Roc de Marsal (d'après Karnay et al. 1999). Figure 3 - Simplified geological map and location of the Roc de Marsal site (adapted from Karanay et al. 1999).

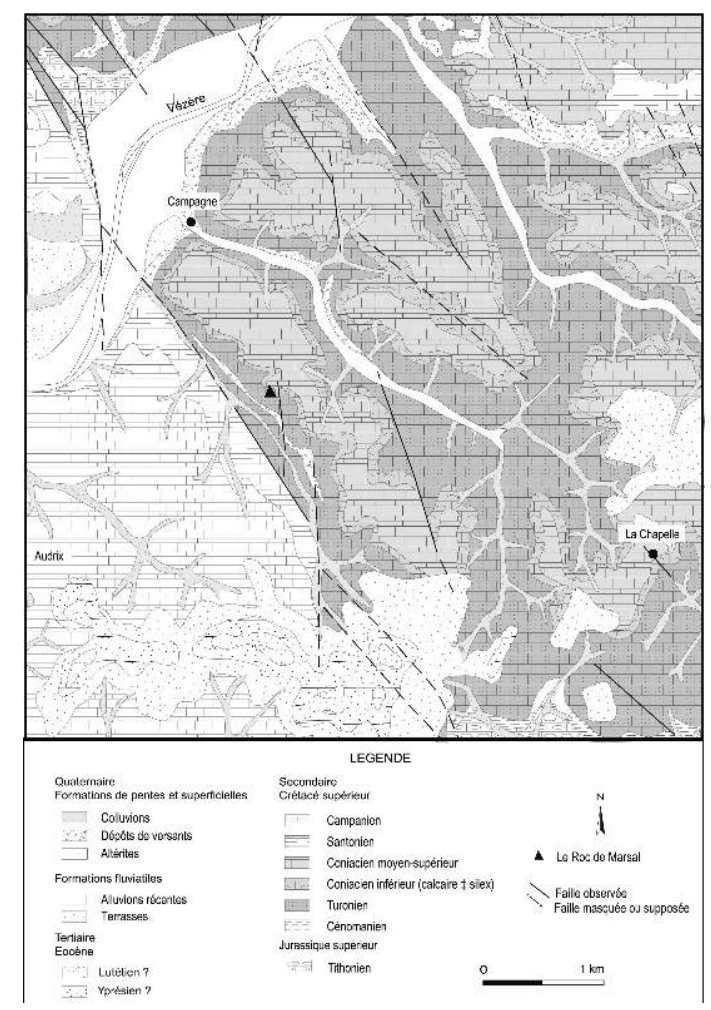

\section{Les matières premières}

Ce travail a été effectué en collaboration avec A. Morala et nous reprenons ici les observations effectuées lors de notre travail de maîtrise (Thiébaut 2000).

À l'exception d'un percuteur en quartz, cette industrie est composée uniquement de vestiges lithiques en silex et presque exclusivement du silex du Sénonien local (de $97,7 \%$ à $100 \%$ selon les catégories). Bien qu'il soit assez difficile de distinguer au sein de ce groupe les différents sous-étages, on peut avancer la prédominance du silex coniacien et la présence très minoritaire du silex du Campanien et du Santonien.

Le gîte de silex coniacien le plus proche se situe au-dessus du gisement, ce qui explique son utilisation privilégiée. À l'intérieur de cet ensemble sénonien, le silex noir et gris 
domine très largement le silex blond. Cependant, la présence d'objets lithiques de couleur mixte (gris et blond, de 4 à $9 \%$ ), témoigne d'un mélange à l'intérieur d'un même bloc de silex blond et de silex noir du Sénonien. Nous pouvons aussi noter la présence de silex blond et gris à zone sous-corticale rouge. En ce qui concerne les silex blonds, ils ont été identifiés depuis plusieurs années (Turq 1977 ; Morala 1980 ; Demars 1980) et sont issus des altérites crétacées. Pour ce qui est des silex gris, ils se localisent dans les bancs de silex à pyrite reconnu dans le Sarladais (Turq et al. 1999).

Parallèlement à cette production de supports en matière première locale (présente dans toutes les catégories technologiques de vestiges), certaines pièces attestent l'utilisation de matières premières allochtones: silex du Bergeracois et calcédoine probablement tertiaire en très faible proportion ( $1,5 \%$ maximum pour le silex maestrichtien et $0,7 \%$ maximum pour la calcédoine tertiaire). Cependant, il s'agit exclusivement de produits finis (éclats Levallois, éclats bruts ou retouchés).

La présence de pièces présentant un cortex néo-fluviatile au sein des différentes catégories technologiques et d'un percuteur en quartz témoigne de l'exploitation des ressources alluviales.

Cette étude indique donc quatre origines différentes des matières premières utilisées :

- un territoire proche du gisement : le plateau même sur lequel est implanté le site (silex du Coniacien) et les collines qui lui font face (silex du Campanien et du Santonien) dont la matière première est importée sous la forme de blocs bruts et testés ;

- deux territoires plus éloignés : l'un à l'ouest dans la région de Bergerac (silex du Maestrichtien) et l'autre en rive gauche de la Dordogne dont le gîte le plus proche se situe au lieu-dit La Bessède (Calcédoine, Turq 2000) dont la matière première est ramenée sous la forme de produits finis.

- enfin, les alluvions de la Vézère ou de la Dordogne pour la recherche de galets de silex et de percuteurs.

\section{Synthèse de l'analyse techno-typologique}

La composition de l'industrie, qui compte 4021 pièces, est présentée dans le tableau cidessous (tabl. 2) :

Tableau 2 : Composition de l'industrie lithique du Roc de Marsal (couche III). Table 2 - Composition of the Roc de Marsal industry (layer III).

\begin{tabular}{|l|l|l|}
\hline & $\mathrm{n}=$ & $\%$ \\
\hline \hline percuteurs & 1 & 0,03 \\
\hline \hline blocs bruts ou testés & 2 & 0,05 \\
\hline \hline nucléus & 140 & 3,5 \\
\hline éclats bruts & 1268 & 31,5 \\
\hline \hline éclats retouchés & 185 & 4,6 \\
\hline fragments d'éclats bruts & 829 & 20,6 \\
\hline
\end{tabular}




\begin{tabular}{|l|l|l|}
\hline éclats $<30 \mathrm{~mm}$ & 903 & 22,5 \\
\hline éclats brûlés non étudiés & 691 & 17,2 \\
\hline fragments de bifaces ?* & 2 & 0,05 \\
\hline Total & $\mathbf{4 0 2 1}$ & 100 \\
\hline
\end{tabular}

* Il s'agit probablement de deux fragments distaux de bifaces décomptés précédemment comme un fragment distal de racloir convergent et de denticulé.

\section{Le débitage}

$\mathrm{Au}$ sein de cette industrie, plusieurs méthodes de débitage ont été reconnues pour la production de supports bruts : Discoïde, Kombewa et Levallois.

Le débitage Discoïde est représenté par près de 50 nucléus (fig. 4), soit presque $35 \%$ de l'ensemble des nucléus. Ils présentent tous un rythme continu du débitage selon la définition proposée par L. Slimak (Slimak 2003), un plan d'intersection total ou partiel de la surface de débitage et de la surface de plans de frappe. Le débitage s'est effectué à partir de blocs ou d'éclats corticaux épais selon trois modalités différentes de la conception volumétrique du bloc: en premier, une conception unifaciale stricte, ensuite une conception bifaciale hiérarchisée et plus rarement une conception bifaciale alternante. Si l'on considère la direction des enlèvements, là encore plusieurs modalités ont été utilisées en fonction des obstacles techniques et des produits recherchés : la mieux représentée est une modalité strictement centripète des enlèvements (31 nucléus) suivi des modalités de débitage unipolaire, centripète et cordale et très sporadiquement bipolaire. Contrairement à ce qui a été défini (Boëda 1993), le plan de fracturation des enlèvements est, au sein de cette série, majoritairement parallèle et/ ou subparallèle au plan d'intersection des deux surfaces. En effet, seulement 16 nucléus présentent des plans de fracturation sécants au plan d'intersection des deux surfaces. Cette observation rejoint celles de V. Mourre sur les séries de Coudoulous (Mourre 1994, 2003) et de L. Slimak sur certains nucléus Discoïdes du Champ Grand et de Néron (Slimak 1997-1998). Certains nucléus témoignent d'une préparation des plans de frappes (41\%). 
Figure 4 - Roc de Marsal, couche III. Nucléus Discoïdes (silex). Figure 4 - Roc de Marsal, layer III. Discoid cores (flint).
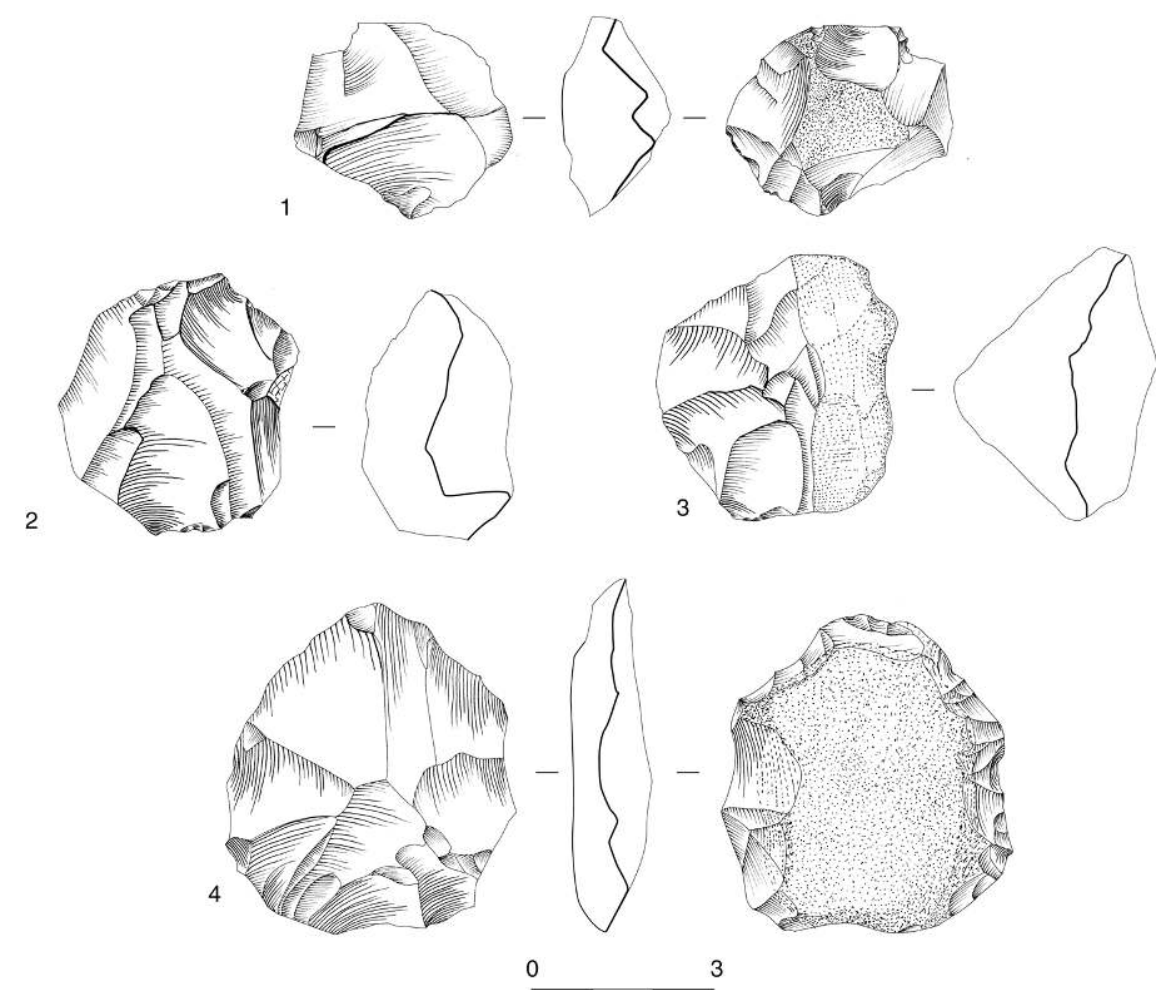

La variabilité des produits obtenus est à corréler avec les différentes modalités utilisées lors du débitage. En effet, d'une manière générale, les produits issus de la modalité centripète seront plutôt courts et larges, tandis que ceux issus des méthodes unipolaires ou bipolaires présenteront des enlèvements plus allongés. En ce qui concerne la présence de plusieurs méthodes de débitage (Levallois, Discoïde, Kombewa), nous ne nous sommes pas risqué à une quantification des différents types de supports produits selon le concept Discoïde. Néanmoins, $37 \%$ des éclats bruts et $32 \%$ des éclats retouchés non-Levallois et non-Kombewa correspondent à une modalité centripète du débitage, tandis que $39 \%$ des éclats bruts présentent une longueur nettement supérieure à leur largeur. Les enlèvements à dos débordant restent minoritaires et ne représentent que $10 \%$ de l'ensemble des éclats ordinaires bruts et retouchés. Notons aussi la présence de quelques éclats pouvant provenir du façonnage de bifaces.

En ce qui concerne le débitage sur éclat au sens strict du terme, deux méthodes semblent coexister au sein de cette industrie : la méthode de type Kombewa (Owen 1938 ; Tixier et Turq 1999) qui prédomine nettement (43 nucléus) et un débitage sur la face supérieure de l'éclat nucléus très peu représenté (2 nucléus). En effet, alors que la très grande majorité des éclats-nucléus présente des négatifs d'enlèvements sur la face inférieure, deux éclats présentent respectivement des enlèvements à partir de la face inférieure et à partir d'une fracture en partie distale. Concernant la méthode de type Kombewa, la conception volumétrique du nucléus est essentiellement unifaciale $(n=$ 34) mais aussi, de manière plus sporadique, bifaciale hiérarchisée $(n=11)$. Le nombre 
d'éclats issus de cette méthode est assez restreint $(n=33)$. Comme pour le débitage Discoïde, le débitage Kombewa a été mis en œuvre selon quatre modalités :

- une modalité linéale avec un axe de débitage de l'éclat Kombewa identique à l'axe de l'éclatnucléus (fig. $5 \mathrm{n}^{\circ} 5$ );

- une modalité linéale dans laquelle l'axe de débitage du produit est différent de celui de l'éclat support ;

- une modalité récurrente unipolaire (fig. $5, \mathrm{n}^{\circ} 6$, et 8 );

- une modalité récurrente bipolaire (fig. $5, \mathrm{n}^{\circ} 4$ et 7 ).

Figure 5 - Roc de Marsal, couche III. $N^{\circ} 1$ à 4, éclats de type Kombewa, ${ }^{\circ} 5$ à 6 , nucléus sur éclats (silex).

Figure 5 - Roc de Marsal, layer III. $N^{\circ} 1$ to 4, Kombewa flakes, $n^{\circ} 5$ to 6 , Kombewa cores (flint).
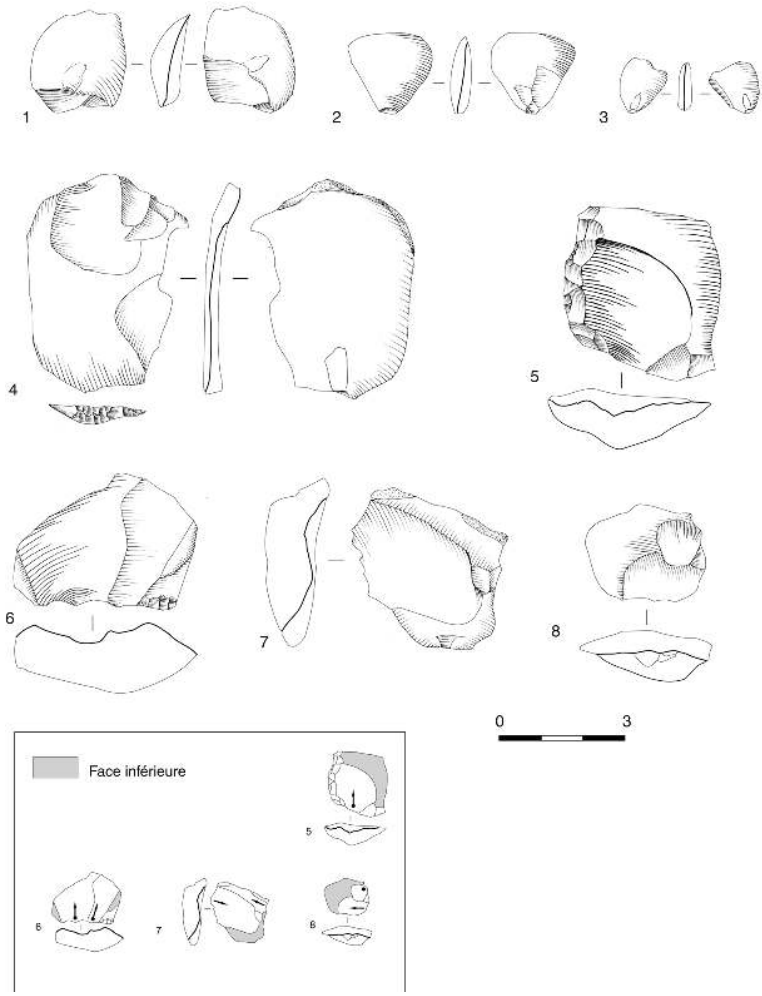

44 Figure 6 - Roc de Marsal couche III. Nucléus Levallois (silex).

Figure 6 - Roc de Marsal, layer III. Levallois cores (flint). 

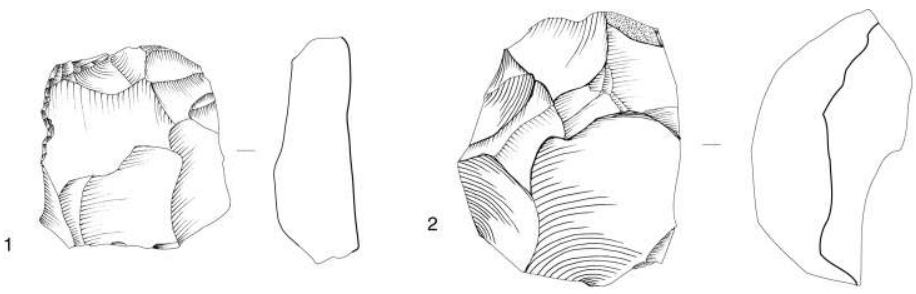

3
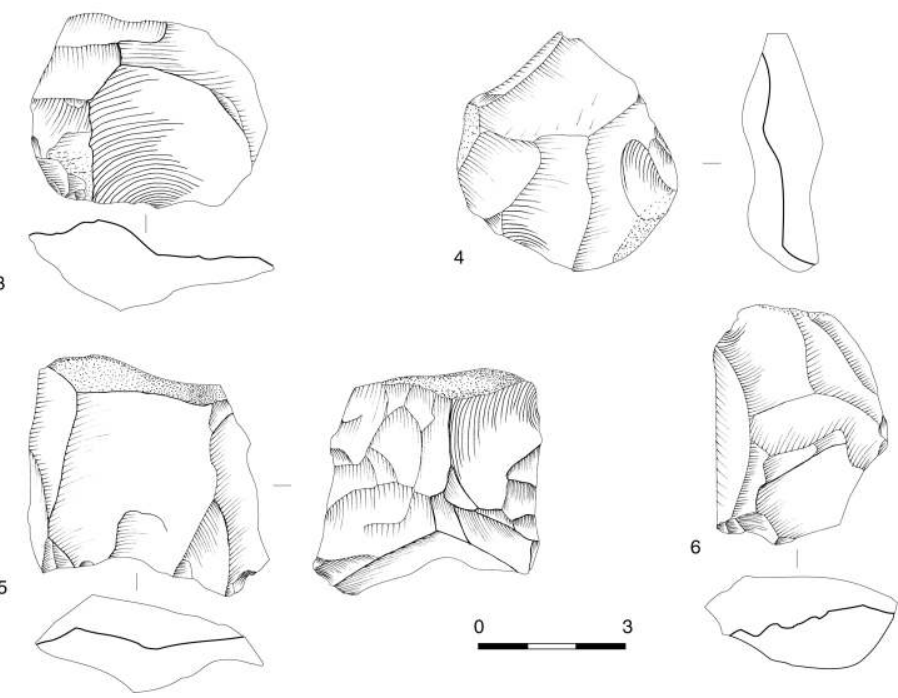

Figure 7 - Roc de Marsal couche III. Éclats Levallois (silex) Figure 7 - Roc de Marsal, layer III. Levallois flakes (flint).
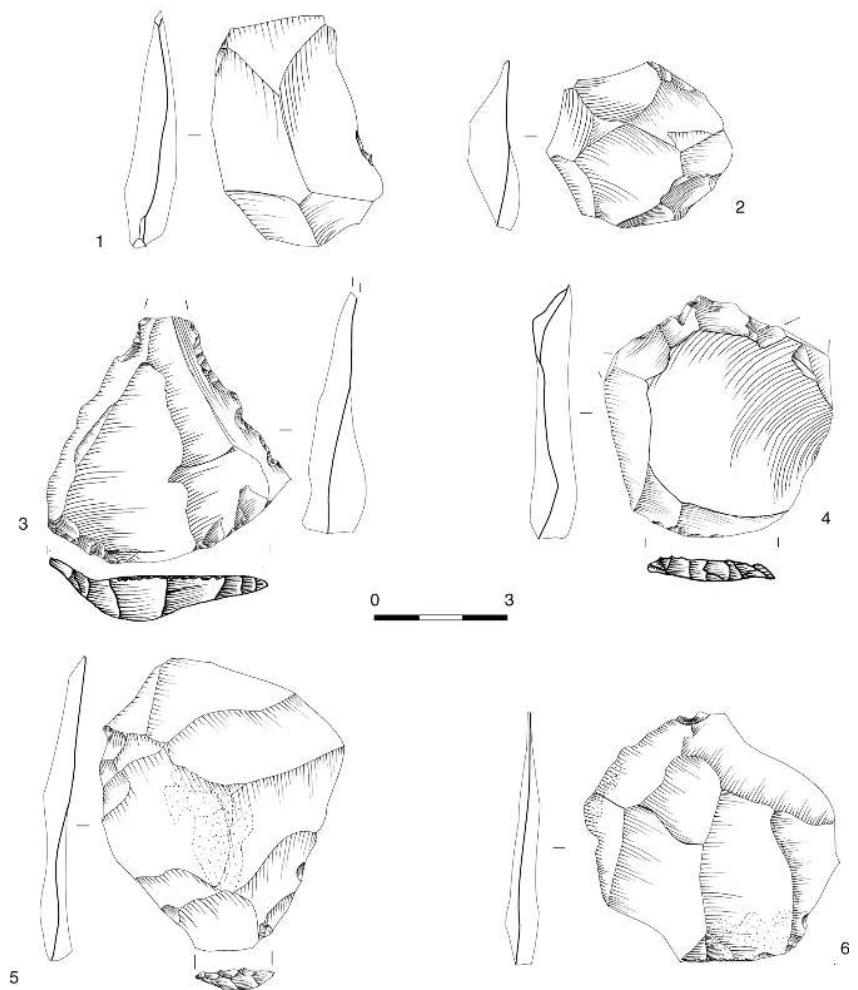
Figure 8 - Différents types de négatifs d'enlèvements (d'après les données de D.-Ch. Prost 1989 et 1993).

Figure 8 - Different types of flaking scars (adapted from D.-Ch. Prost 1989 and 1993).

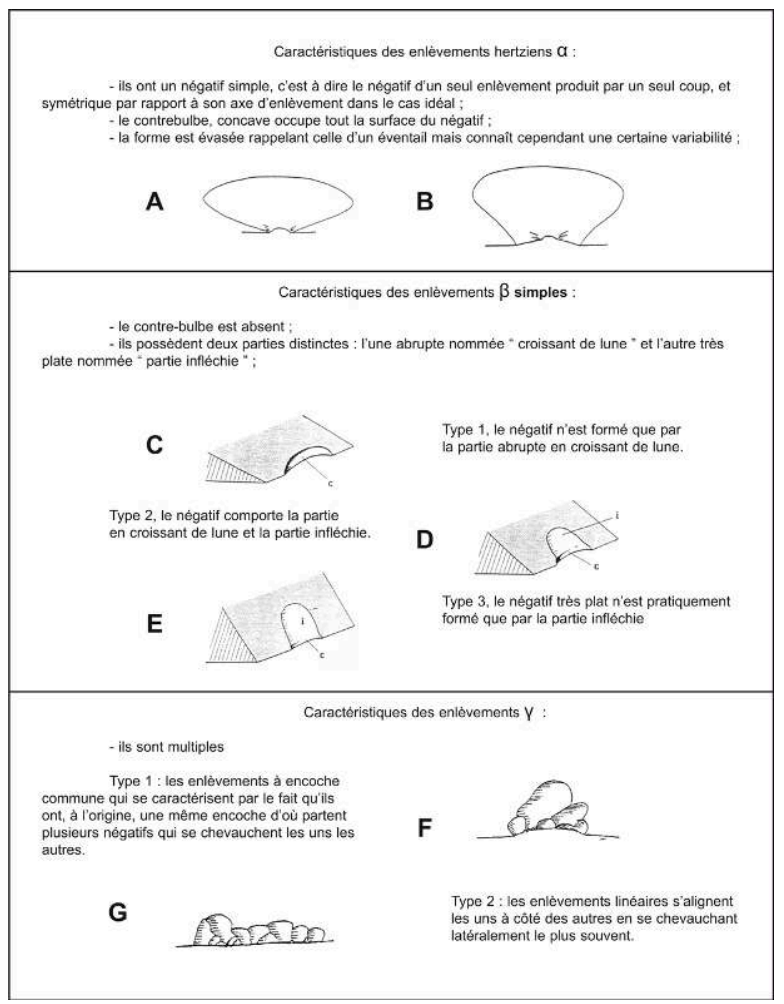

Ces différentes modalités entraînent une certaine variabilité des produits obtenus : des éclats de type Kombewa Janus (fig. $5, \mathrm{n}^{\circ} 2$ et 3 ), des éclats de type Kombewa ordinaires (fig. $5 \mathrm{n}^{\circ}$ 4) et des éclats de type Kombewa à dos débordants (fig. $5 \mathrm{n}^{\circ} 8$ ).

Le concept de débitage Levallois est illustré essentiellement par la présence d'éclats Levallois typiques. La faiblesse numérique des nucléus Levallois $(n=17)$ est, selon nous, imputable à l'état d'exhaustion des nucléus parfois repris selon un concept Discoïde. L'analyse des nucléus témoigne d'une prédominance de la méthode linéale du débitage (fig. 6, $n^{\circ} 2,3$ et 5). Cependant, l'état exhaustif des nucléus, souvent atypiques, reste un obstacle lors de leur attribution aux diverses méthodes existantes (fig. $6, \mathrm{n}^{\circ} 2$ et 6 ).

Bien que très peu représentée, la méthode récurrente $(n=5)$ est présente selon des modalités centripète, unipolaire ou bipolaire (fig. $6, \mathrm{n}^{\circ} 1,4$ et 6 ). Plus que les nucléus, ce sont les éclats Levallois $(n=117)$ qui nous ont permis de mettre en valeur la présence majoritaire d'une méthode linéale du débitage. En effet, les éclats Levallois premiers (issus d'une modalité linéale ou récurrente) représentent plus de $74 \%$ de l'ensemble des éclats Levallois (fig. $7 \mathrm{n}^{\circ} 1,3,5,6$ ), tandis que les éclats seconds et troisièmes représentent, respectivement, près de $17 \%$ et $9 \%$ (fig. $7 \mathrm{n}^{\circ} 4$ ). Ils sont principalement de morphologie quadrangulaire et ovalaire et plus rarement triangulaire $(10 \%)$. Leur talon est facetté pour une grande majorité d'entre eux, lisse pour seulement $20 \%$ et dièdre ou punctiforme pour $16,5 \%$ et $5 \%$.

\section{L'outillage retouché}

Au sein des 271 pièces considérées comme retouchées, l'ensemble du matériel lithique, tous types de supports confondus, atteint désormais $9,2 \%(n=210)$ de l'ensemble des 
produits de débitage (éclats brûlés non étudiés exclus) si l'on ne considère plus les pseudo-outils et $8,1 \%(\mathrm{n}=185)$ si l'on ne considère plus les pseudo-outils probables (tabl. 3).

Tableau 3 - Roc de Marsal couche III. Composition de l'outillage retouché en 2000 et 20002. Table 3 - Roc de Marsal, layer III. Composition of the flake tools group.

\begin{tabular}{|c|c|c|c|c|c|c|}
\hline \multicolumn{3}{|c|}{2000} & \multicolumn{4}{|c|}{ juin 2002} \\
\hline Outils retouchés & $\mathrm{n}=$ & $\%$ & $\begin{array}{c}\text { sans } \\
\text { pseudo } \\
n=\end{array}$ & $\%$ & $\begin{array}{c}\text { sans les } \\
\text { pseudo } \\
\text { probable } \\
\mathrm{n}=\end{array}$ & $\%$ \\
\hline Racloirs & 66 & 24,4 & 70 & 33,0 & 70 & 37,4 \\
\hline simpl.droit & 26 & 9,6 & 27 & 12,7 & 27 & 14,4 \\
\hline simpl. Convexe & 21 & 7,7 & 22 & 10,4 & 22 & 11,8 \\
\hline dbl.droit & 3 & 1,1 & 3 & 1,4 & 3 & 1,6 \\
\hline dbl.droit/convexe & 1 & 0,4 & 2 & 0,9 & 2 & 1,1 \\
\hline dbl.biconvexe & 1 & 0,4 & 1 & 0,5 & 1 & 0,5 \\
\hline converg. droit & 4 & 1,5 & 3 & 1,9 & 3 & 2,1 \\
\hline converg. convexe & 3 & 1,1 & 3 & 1,4 & 3 & 1,6 \\
\hline déjeté & 1 & 0,4 & 1 & 0,5 & 1 & 0,5 \\
\hline transver. droit & 3 & 1,1 & 3 & 1,4 & 3 & 1,6 \\
\hline transver. convexe & 2 & 0,7 & 2 & 0,9 & 2 & 1,1 \\
\hline ret. Alterne & 1 & 0,4 & 2 & 0,9 & 2 & 1,1 \\
\hline Grattoirs & 11 & 4,1 & 9 & 4,2 & 9 & 4,8 \\
\hline typique & 1 & 0,4 & 1 & 0,5 & 1 & 0,5 \\
\hline atypiques & 10 & 3,7 & 8 & 3,9 & 8 & 4,3 \\
\hline Burin & 1 & 0,4 & 1 & 0,5 & 1 & 0,5 \\
\hline typique & 1 & 0,4 & 1 & 0,5 & 1 & 0,5 \\
\hline Éclats tronqués & 4 & 1,5 & 3 & 1,4 & 2 & 1,1 \\
\hline Encoches & 65 & 24,0 & 40 & 18,9 & 35 & 18,7 \\
\hline Denticulés & 47 & 17,3 & 33 & 16,0 & 27 & 15,0 \\
\hline Pics & 6 & 2,2 & 5 & 2,4 & 5 & 2,7 \\
\hline Amincis & 10 & 3,7 & 10 & 4,7 & 10 & 5,3 \\
\hline Divers & 40 & 14,8 & 30 & 14,2 & 17 & 9,1 \\
\hline Composites & 22 & 8,1 & 10 & $\mathbf{4 , 7}$ & 10 & 5,3 \\
\hline Total & 271 & 100 & 210 & 100 & 185 & 100 \\
\hline
\end{tabular}

\begin{tabular}{|c|c|c|}
\hline Composites & 2000 & $\begin{array}{l}\text { juin } \\
\mathbf{2 0 0 2}\end{array}$ \\
\hline $\begin{array}{l}\text { rac.simpl.dt } \\
\text { /encoche }\end{array}$ & 3 & 2 \\
\hline $\begin{array}{l}\text { rac.simpl.dt } \\
\text { /denti. }\end{array}$ & 1 & 1 \\
\hline $\begin{array}{l}\text { racl.simpl.cx } \\
\text { /encoche }\end{array}$ & 1 & 1 \\
\hline $\begin{array}{l}\text { racl.simpl.cx } \\
\text { /pic }\end{array}$ & 1 & 0 \\
\hline $\begin{array}{l}\text { racl. ret alt. } \\
\text { /encoche }\end{array}$ & 1 & 0 \\
\hline encoche /tronc. & 1 & 0 \\
\hline double encoche & 3 & 1 \\
\hline encoche /denti. & 2 & 1 \\
\hline $\begin{array}{c}\text { encoche } \\
\text { /denti. /tronc }\end{array}$ & 1 & 1 \\
\hline encoche/pic & 2 & 0 \\
\hline $\begin{array}{l}\text { encoche } \\
\text { /divers }\end{array}$ & 3 & 0 \\
\hline denti. /tronc & 3 & 3 \\
\hline Total & 22 & 10 \\
\hline
\end{tabular}

\section{Les pseudo-outils et outils a posteriori}

Les différents phénomènes responsables de la production d'enlèvements naturels ou accidentels comparables à de la retouche anthropique ont fait l'objet de plusieurs publications. Nous distinguerons ici les processus accidentels des processus naturels :

- les enlèvements accidentels sont produits de manière fortuite dans le cadre d'actions anthropiques telles que le débitage (Newcomer 1976 ; Prost 1989), la confection de la retouche (Fournier 1973 ; Prost 1989 ; Thiébaut 2001) et l'utilisation d'un tranchant (Pradel 1956 ; Beyries 1987 ; Prost 1989), le piétinement humain ou animal (Vaufrey 1955 ; Tixier 1958-1959 ; Bordes 1970 ; Tringham et al. 1974 ; Knudson 1979 ; Fleniken et Haggarty 1979 ; Prost 1989 ; Mc Brearty et al. 1998) et les travaux agricoles sur les sites de plein air (Mallouf 1982 ; Prost 1989) ;

- les enlèvements résultent de processus naturels : la pression dans les sols, le ressac, l'action des glaciers, la cryoturbation, la solifluxion (Pei 1936 ; Barnes 1938 ; Breuil et Lantier 1951 ; Bourgon 1957 ; Bordes 1961 ; Bordes 1962-1963).

Tous ces phénomènes sont susceptibles d'altérer les vestiges lithiques et de produire des pseudo-outils. L'état général des pièces (état de surface et du tranchant) permet dans une certaine mesure d'identifier les vestiges susceptibles d'avoir été endommagés. Pour une meilleure analyse, nous proposons un tableau (non exhaustif) issu des travaux 
cités précédemment sur les divers types d'altérations produites par les phénomènes naturels (tabl. 4).

Tableau 4 - Synthèse des stigmates des principaux agents d'altération.

Table 4 - Synthetic presentation of major alteration agents marks.

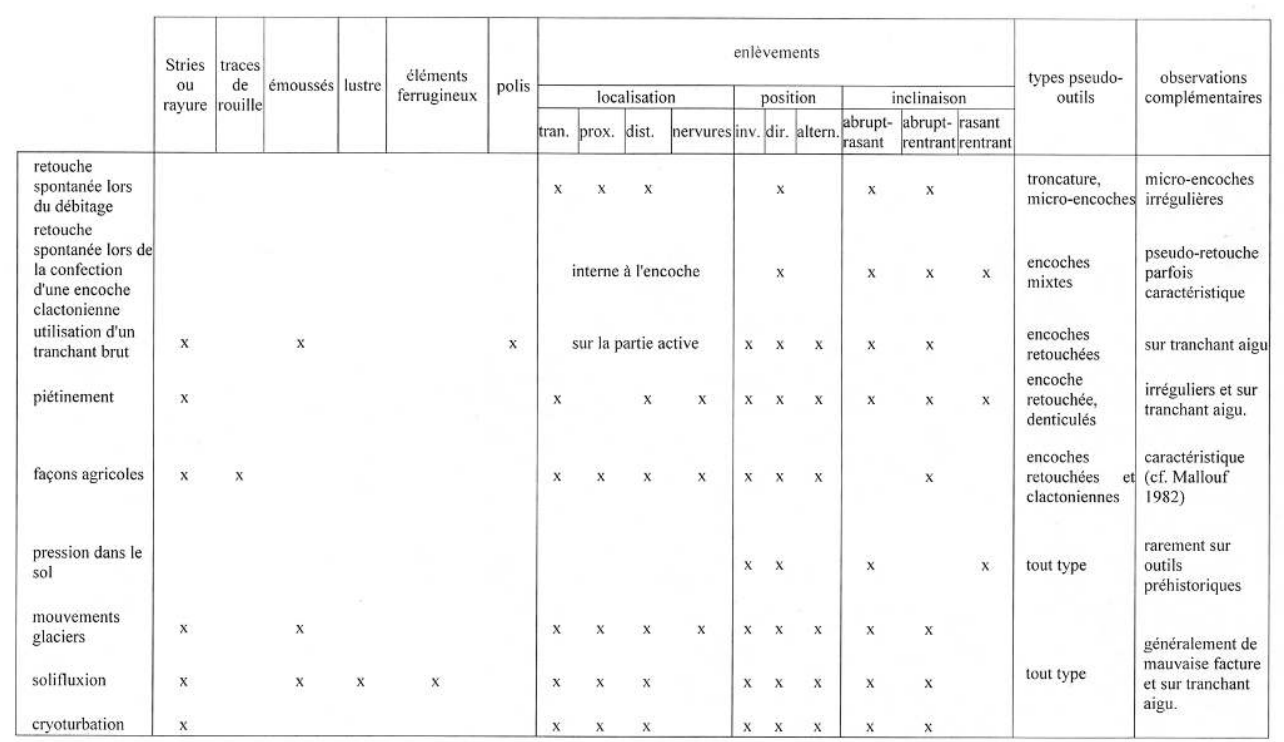

La distinction s'effectuera ensuite par l'observation des types d'encoches et de denticulations présentes sur les vestiges archéologiques et leur comparaison avec ceux issus des diverses expérimentations. Deux listes pourront alors être établies, l'une pour les pièces encochées et la seconde pour les pseudo-outils potentiels. Cette démarche n'est pas novatrice: dès 1970, F. Bordes préconisait la création de deux listes “ typologiques " au travers desquelles on séparerait les outils retouchés et les pseudooutils (Bordes 1970). La description précise des supports et des enlèvements des pièces comptabilisées comme pseudo-outils permettra peut-être de trouver des critères distinctifs qui leur sont propre.

Il n'est ici question que des objets encochés qui présentent une certaine ambiguïté dans l'origine de leur confection. Les denticulés à macrodenticulations ne présentent généralement aucune ambiguïté quant au caractère anthropique des encoches, excepté toutefois les denticulés à macro-encoches d'utilisation. Pour ces derniers, ce sont donc les caractéristiques des encoches qui devront être étudiées.

La reconnaissance typologique des denticulés à microdenticulations présente plus de difficultés. Cependant, il semblerait que deux phénomènes seulement puissent produire ce type de pièce : le piétinement et le concassage. Or, dans les deux cas, la régularité des enlèvements et l'angle $\mathrm{du}$ tranchant peuvent permettre une distinction plus catégorique entre les denticulés à microdenticulations et les pseudo-denticulés.

La reconnaissance formelle des encoches comme objets façonnés par l'homme est plus incertaine. Toutefois, il semblerait que les micro-encoches clactoniennes isolées (ouverture $<5 \mathrm{~mm}$ ) puissent être produites par une grande partie des phénomènes accidentels et naturels cités plus haut. Elles ne seront donc pas comptabilisées ici comme anthropiques. En ce qui concerne les macro-encoches clactoniennes, celles présentant un négatif de type (fig. 8) ou un négatif présentant les caractéristiques d'une flexion peuvent être considérées comme non anthropiques. Pour ce qui est des 
encoches retouchées, la distinction semble moins évidente. Cependant, une encoche retouchée très ouverte et peu profonde dont la retouche est plutôt rasante et sans aspérités localisée dans la concavité et sur les bords caractérise généralement une encoche a posteriori (Thiébaut 2001).

Afin de distinguer les pseudo-outils au sein des pièces retouchées, différentes caractéristiques des tranchants ont été prises en considération :

- la régularité, la continuité et l'inclinaison de la retouche;

- l'angle du tranchant ;

- le caractère général du tranchant et du support ;

- les caractéristiques des négatifs (Prost 1989, fig. 8);

- les caractéristiques morphométriques des encoches (longueur d'ouverture, morphologie, Girard 1978, Thiébaut 2001). les subdiviser en quatre groupes :

- groupe 1 : l'ouverture de l'encoche est inférieure ou égale à $5 \mathrm{~mm}$ et leur support présente un tranchant aigu $\left(<30^{\circ}\right)$ à l'endroit où a été produite l'encoche $(\mathrm{n}=9)\left(\right.$ fig. $\left.9, \mathrm{n}^{\circ} 5\right)$ et deux d'entre elles présentent des enlèvements courts et abrupts à l'intérieur d'un enlèvement de type , ß1 (fracture probable);

- groupe 2 : elles sont peu profondes et présentent des esquillements alternants sur un tranchant dont l'angle est rarement supérieur à $30^{\circ}(\mathrm{n}=4)$;

- groupe 3 : la retouche est irrégulière, souvent peu étendue $(n=1)$, de morphologie ouverte mais peu profonde et sans aspérité au sein de l'encoche $(n=3)$, caractéristiques qui suggèrent des encoches a posteriori (action de translation transversale sur support dur) (fig. $\left.9, \mathrm{n}^{\circ} 10\right)$;

- groupe 4 : la retouche est irrégulière, l'angle du tranchant est aigu mais la présence d'aspérités au sein des encoches suggère une origine liée à un concassage $(n=4)\left(\right.$ fig.10; ${ }^{\circ} 6$ et 11). 
Figure 9 - Roc de Marsal couche III. Pseudo-encoches, sauf $n^{\circ} 9$ pseudo-denticulé (silex). Figure 9 - Roc de Marsal, layer III. Pseudo-notches, except $n^{\circ} 9$ pseudo-denticulate (flint).
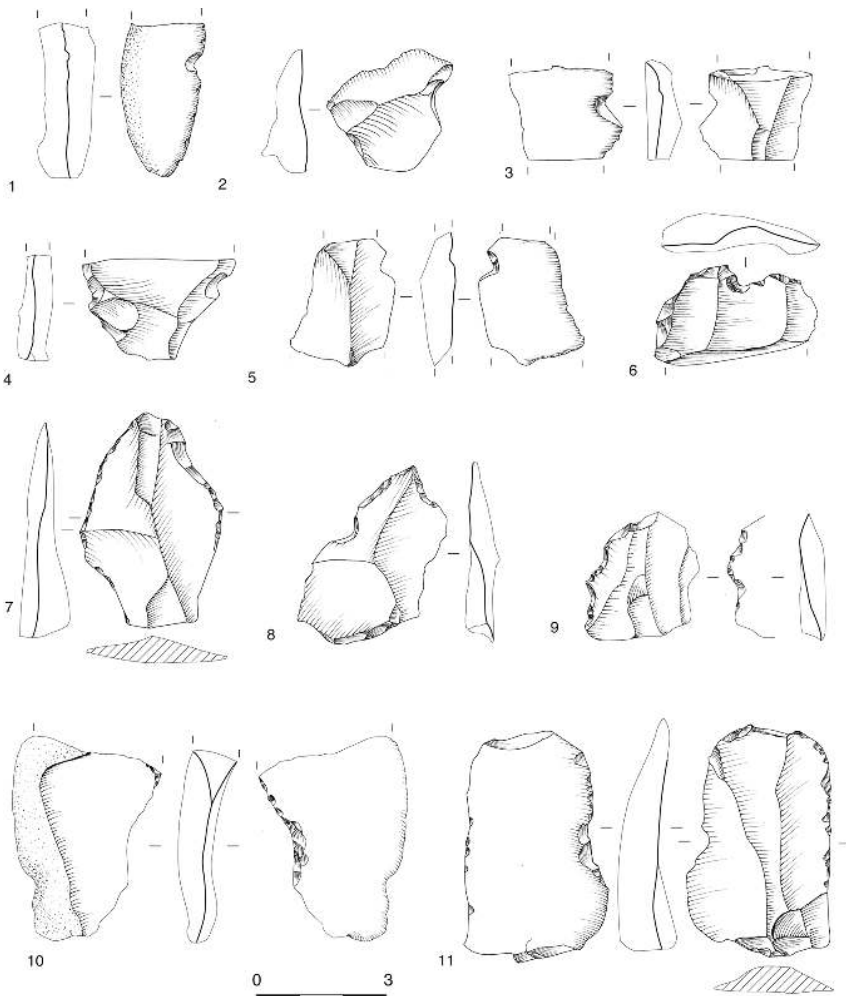

Figure 10 - Roc de Marsal couche III. Pseudo-denticulés (silex) Figure 10 - Roc de Marsal, layer III. Pseudo-denticulates (flint).
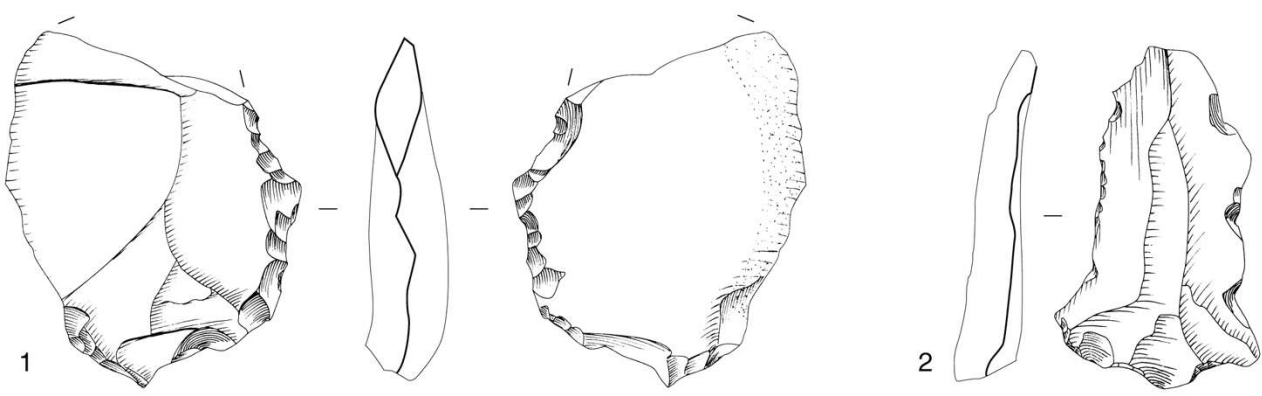

3

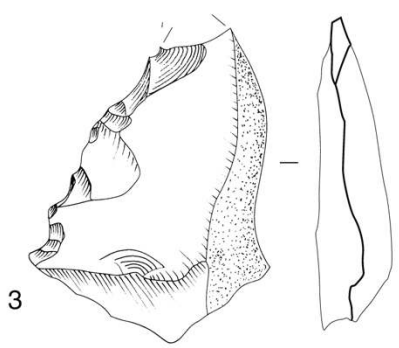

4
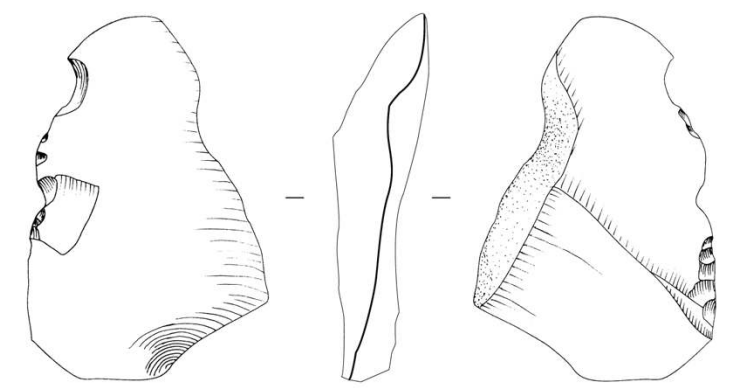
Figure 11 - Roc de Marsal couche III. $N^{\circ} 1$ à 2 encoches, $n^{\circ} 3$ encoche et racloir, $n^{\circ} 4$ à 7 et 9 denticulés, $n^{\circ} 8$ denticulé bec (silex).

Figure 11 - Roc de Marsal, layer III. $n^{\circ} 1$ and 2, notches, $n^{\circ} 3$ notch and scraper, $n^{\circ} 4$ to 7 and 9 denticulates, $n^{\circ} 8$ "bec" denticulate (flint).

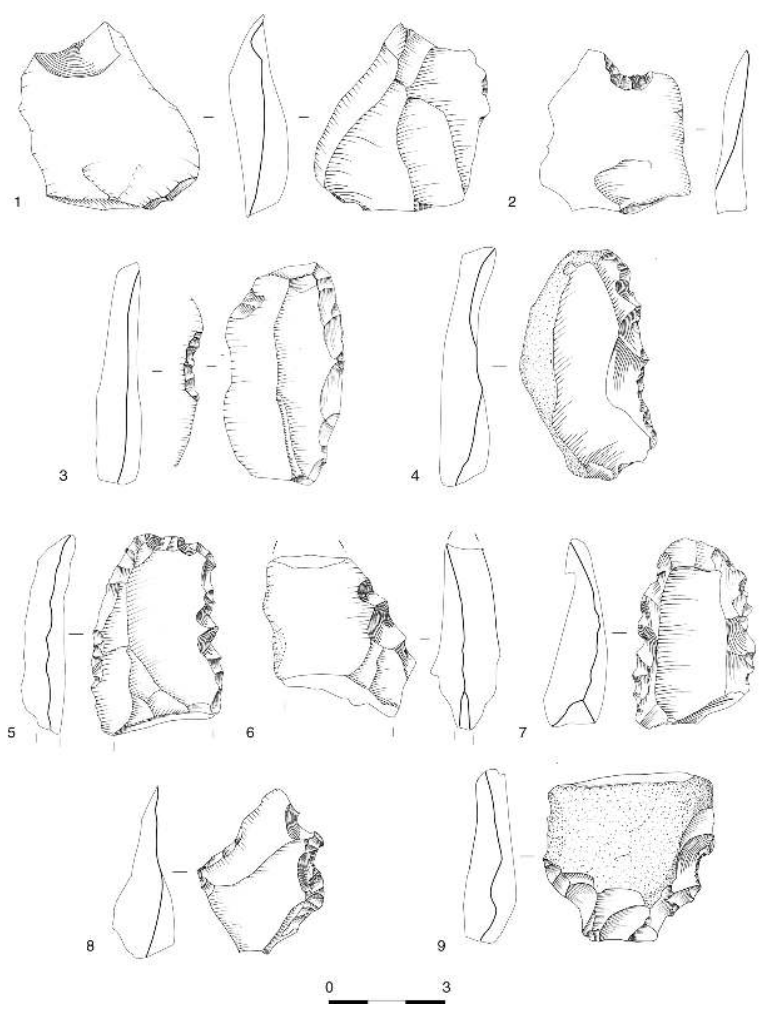

En ce qui concerne les pseudo-encoches probables $(n=3+2)$, le caractère naturel ou accidentel semble moins évident, mais elles apparaissent peu typiques : rasantes et peu concaves pour deux encoches simples, suggérant davantage un enlèvement par flexion pour la troisième et une retouche irrégulière parfois alternante sur des tranchants très aigus pour les deux encoches comptabilisées comme retouchées.

Les critères dominants retenus pour distinguer les pseudo-denticulés et les pseudodenticulés probables $(n=13+6)$ sont de plusieurs ordres. Tout d'abord, le manque de régularité des encoches est quasiment présent sur l'ensemble des pseudo-denticulés, la discontinuité de la retouche associée parfois à la présence d'autres négatifs alternants sur le tranchant, au caractère parfois très abrupt et irrégulier des négatifs d'enlèvements (enlèvements $\alpha$ et $B 1$ ) et à un tranchant relativement aigu. Nous pouvons ainsi subdiviser les pseudo-denticulés en 2 groupes :

- groupe 1 : retouche irrégulière $\left(n=10+3\right.$ pseudo probable, fig. $\left.10, n^{\circ} 3\right)$, parfois discontinue $\left(\mathrm{n}=4\right.$, fig. $\left.10, \mathrm{n}^{\circ} 2\right)$, alternante $\left(\mathrm{n}=2\right.$ fig. $\left.9 \mathrm{n}^{\circ} 9\right)$, discontinue et alternante $(\mathrm{n}=2$,$) associé à$ une double patine $\left(n=2\right.$, fig. $\left.10, n^{\circ} 1\right)$. Plus que le caractère anthropique de la retouche, c'est ici l'attribution typologique de certaines de ces pièces à des denticulés qui est remis en cause ;

- groupe 2 : les caractéristiques des négatifs rappellent celles des cassures de type 11 ou de concassage $(\alpha 1)(n=3+3)$ parfois associé à une retouche discontinue $(n=2)$ ou alternante $(n=2)$ et pour l'un d'entre eux à la fois discontinue et alternante (fig. 10, $\left.n^{\circ} 4\right)$.

Certains des outils dits " composites " et notamment ceux qui présentaient une encoche associée à un racloir ont été rattachés au groupe des racloirs simples. 


\section{Les principales catégories typologiques} considérés comme anthropiques, nous pouvons constater que d'un point de vue technologique et quelle que soit la catégorie typologique des outils, ce sont les éclats ordinaires qui ont été principalement utilisés pour la confection des pièces encochées et des racloirs. Néanmoins, en ce qui concerne les denticulés il semblerait que les supports à dos (de débitage ou corticaux) aient été légèrement privilégiés par rapport aux éclats ordinaires $(n=14)($ tabl. 5).

Tabeau 5 - Roc de Marsal, couche III. Répartition des principaux outils par types de supports. Table 5 - Roc de Marsal, layer III. Repartition of the major tool-classes according to support types.

\begin{tabular}{|l|ccc|c|}
\hline \multicolumn{1}{|c|}{ Supports } & encoches & denticulés & racloirs & Total \\
\hline entame & 0 & 0 & 1 & 1 \\
éclat à dos débordant & 4 & $\mathbf{5}$ & 5 & 14 \\
éclat dos cortical & 4 & $\mathbf{6}$ & 5 & 15 \\
éclat cortical & 2 & 3 & 5 & 10 \\
éclat ordinaire & $\mathbf{1 5}$ & $\mathbf{8}$ & $\mathbf{3 2}$ & 56 \\
éclat Levallois & 10 & 3 & 16 & 29 \\
éclat laminaire & 0 & 2 & 3 & 5 \\
éclat kombewa & 0 & 0 & 3 & 3 \\
\hline \multicolumn{1}{|c}{ Total } & 35 & 27 & 70 & $\mathbf{1 3 3}$ \\
\hline
\end{tabular}

D'un point de vue typologique, les encoches sont généralement retouchées $(\mathrm{n}=17$, fig. $11, n^{\circ} 2$ et 3$)$, plus rarement clactoniennes $\left(n=9\right.$, fig. $\left.11, n^{\circ} 1\right)$ ou mixtes $(n=4)$.

Les denticulés se subdivisent équitablement entre denticulés à macrodenticulations et denticulés à microdenticulations $(\mathrm{n}=14$ et 14$)$. Ces derniers sont confectionnés par de petits enlèvements de retouche qui créent des microdenticulations (fig. $11, \mathrm{n}^{\circ} 5$ et 7 ). Les autres denticulés ont été produits par de grandes encoches simples $(n=7)$, retouchées $(n=6)$ ou mixtes $(n=1)$ créant des denticulés à macrodenticulation (fig. 11 $n^{\circ} 4$ ) et moyenne denticulation (fig. $11, n^{\circ} 6$ ). Certains d'entre eux sont cependant peu typiques (fig. 11, $\mathrm{n}^{\circ}$ 9) ou tendant vers le bec (fig. 11, $\mathrm{n}^{\circ} 8$ ).

e manière générale, mis à part un petit nombre de denticulés, cette industrie ne présente pas un nombre important d'outils encochés typiques tels que ceux que l'on retrouve au sein d'autres séries lithiques rattachées au Moustérien à denticulés comme celles de Mauran (Farizy et al. 1994), de La Borde (Jaubert et al.1990), de l'Hyène couche VIb1 (Girard 1978, Thiébaut en cours), du Bison couche G (David et al. 2002) et qui dépassent très nettement le groupe des racloirs. En effet, que ce soit à Mauran ou La Borde, les denticulés forment un groupe important, typique, très diversifié et cela, quelle que soit la matière première utilisée.

Si nous nous référons aux pièces encochées de Mauran, trois observations doivent être retenues :

- la prédominance des denticulés et des encoches $(n=70)$ sur les racloirs $(n=21)$;

- la nette prédominance des encoches clactoniennes (fig. $12, \mathrm{n}^{\circ} 2,8$ et 10 ) sur les encoches retouchées $\left(n=19\right.$ et 1 , fig. $\left.12 n^{\circ} 4\right)$; 
- la grande diversité des denticulés : présence de denticulés à macrodenticulation (fig. 12, $\mathrm{n}^{\circ} 3,6,11,12$ et 13 ), de denticulés à moyenne-denticulation (fig. 12, $\mathrm{n}^{\circ} 5,7$ et 9) et de denticulés à microdenticulation (fig. 12, $\mathrm{n}^{\circ} 1$ ).

Figure 12 - Mauran. $N^{\circ} 1,9,11$ et 12 denticulés (quartzite), $n^{\circ} 3,5,6$ et 7 denticulés (silex), $n^{\circ} 13$, denticulé (quartz), $n^{\circ} 2,4$ et 10 encoches (silex), $n^{\circ} 8$ encoche (quartzite) (Jaubert in Farizy, David, Jaubert (dir.) 1994).

Figure 12 - Mauran. $N^{\circ} 1,9,11$ and 12 denticulates (quartzite), $n^{\circ} 3,5,6$ and 7 denticulates (silex), $n^{\circ} 13$, denticulate (quartz), $n^{\circ} 2,4$ and 10 notches (silex), $n^{\circ} 8$ notch (quartzite) (Jaubert in Farizy, David, Jaubert (dir.) 1994).

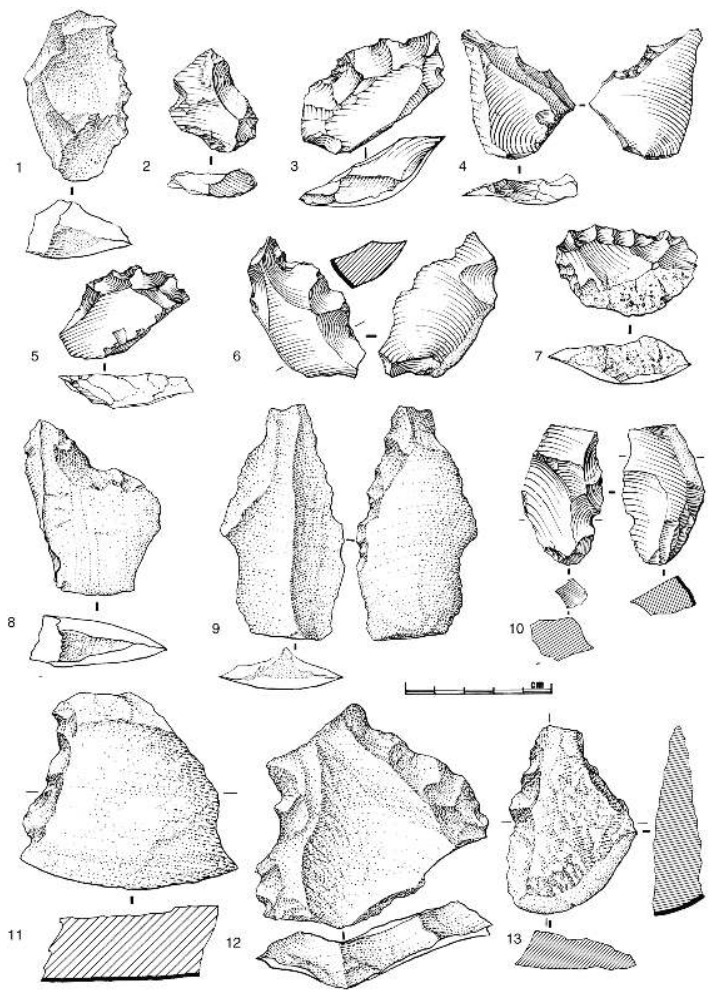

Au sein de la série de La Borde, bien que les racloirs représentent 23, $2 \%$ de l'ensemble des outils retouchés $(n=81)$, certains d'entre eux présentent une retouche proche d'une denticulation (comme le remarque J. Jaubert, (Jaubert et al. $1990 ;$ p. 83 et 91 et fig. $45 \mathrm{n}^{\circ} 3,4,5,6$ et 7 et fig. $62, \mathrm{n}^{\circ} 1$ et 2). De plus, le groupe des pièces encochées reste nettement dominant $(n=156)$ puisqu'il représente $46 \%$ des outils retouchés. Là encore les encoches clactoniennes semblent plus fréquentes (Jaubert et al. 1990, fig. 13, n 1 à 3) et les denticulés sont très diversifiés : denticulés à macro-encoches (fig. $13, \mathrm{n}^{\circ} 7,8,9$ et 10) denticulés à moyennes encoches (fig. $12 n^{\circ} 4$ et 6 ) et denticulés à micro-encoches (fig. 13, $\mathrm{n}^{\circ}$ 5). 
Figure 13 - La Borde. $N^{\circ} 1$ et 2 encoches (silex), $n^{\circ} 3$ encoche (quartz), $n^{\circ} 4$ et 9 denticulés (silex), $n^{\circ} 5$ à 7 denticulés (quartz) (Jaubert in Farizy, David, Jaubert (dir.) 1994).

Figure 13 - La Borde. $N^{\circ} 1$ and 2 notches (silex), $n^{\circ} 3$ notch (quartz), $n^{\circ} 4$ and 9 denticulates (silex), $n^{\circ} 5$ to 7 denticulates (quartz) (Jaubert in Farizy, David, Jaubert (dir.) 1994).
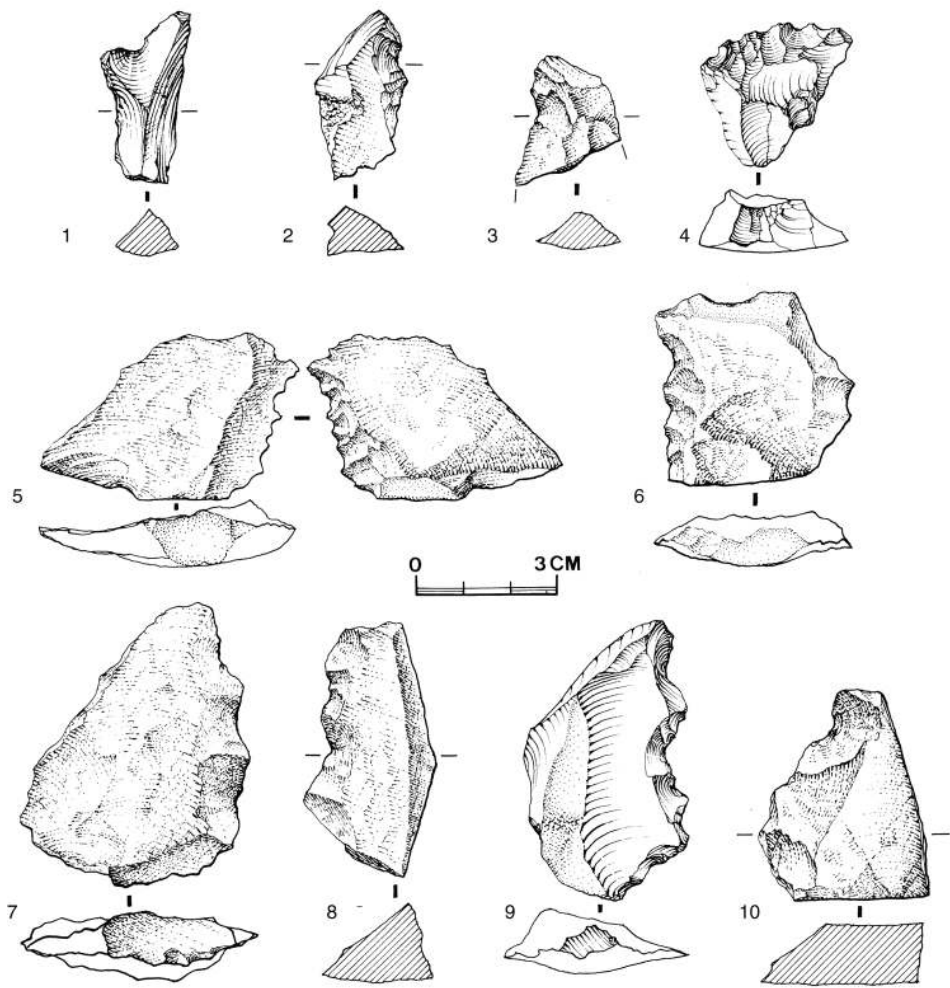

En comptabilisant certaines pièces comme pseudo-pièces encochées, au sein de l'industrie de la couche III du Roc de Marsal, nous remarquons que le groupe des racloirs équivaut ou domine celui des encoches et des denticulés réunis. Cette industrie ne correspond alors plus, en terme statistique, à ce que F. Bordes appelle le Moustérien à denticulés. Nous pouvons aussi remarquer qu'au-delà de la prédominance de la classe des racloirs, ces derniers ne correspondent pas à ce que l'on peut appeler “ des racloirs frustes ". En effet, la retouche est régulière et rasante, jamais alternante, majoritairement continue et totale sur tout le tranchant (fig. 14). Mais peut-être que la bonne ou mauvaise facture des racloirs ne correspond pas véritablement à une caractéristique discriminante lors de l'attribution d'une industrie lithique au Moustérien à denticulés. 
Figure 14 - Roc de Marsal couche III : racloirs sauf $n^{\circ} 9$ grattoir (silex). Figure 14 - Roc de Marsal layer III. Scrapers, except $n^{\circ} 9$ end scraper (flint).
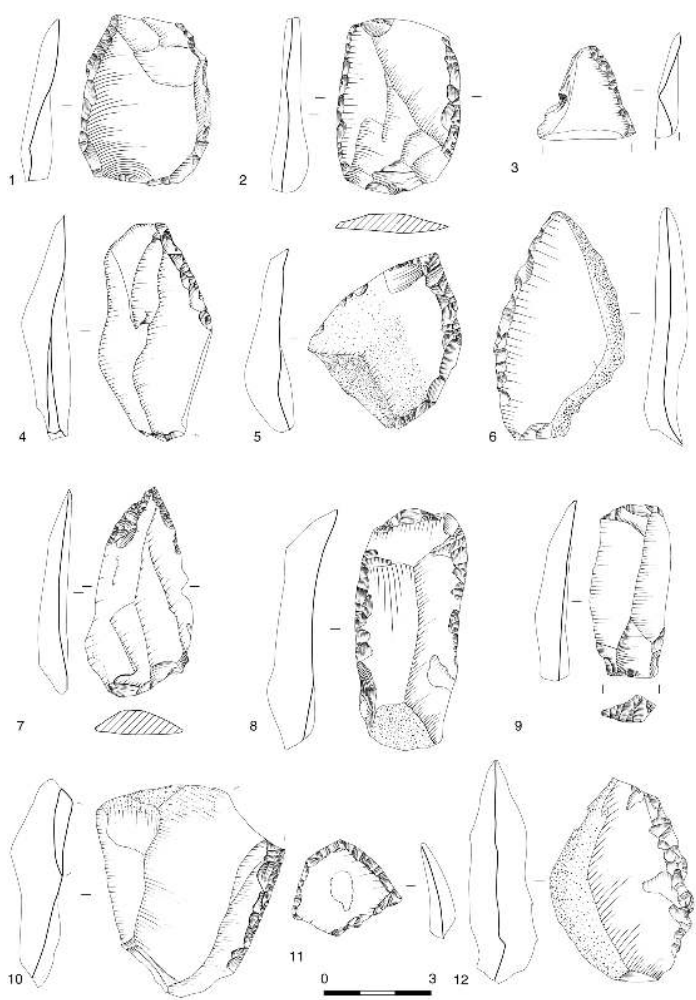

70 Nous pouvons donc contester l'attribution de la série de la couche III du Roc de Marsal au Moustérien à denticulés et nous interroger sur celle de l'industrie lithique de la couche II dont certaines pièces appartenant au groupe des outils encochés paraissent douteuses $^{4}$ (fig. $15, n^{\circ} 1,2,3$, et 15 ) alors que les racloirs sont très bien représentés, avec un indice de 40 en décompte essentiel (Antignac, 1998). De plus, G. Antignac soulignait dès 1998 la présence éventuelle de pseudo-outils au sein de cette série (Antignac 1998; p. 32). 
Figure 15 - Roc de Marsal couche II. $\mathrm{N}^{\circ} 1$ à 6 encoches (silex), $\mathrm{n}^{\circ} 7$ à 15 denticulés (silex) (Antignac, 1998).

Figure 15 - Roc de Marsal layer II. $N^{\circ} 1$ to 6 notches (flint), $n^{\circ} 7$ to 15 denticulates (flint) (Antignac, 1998).

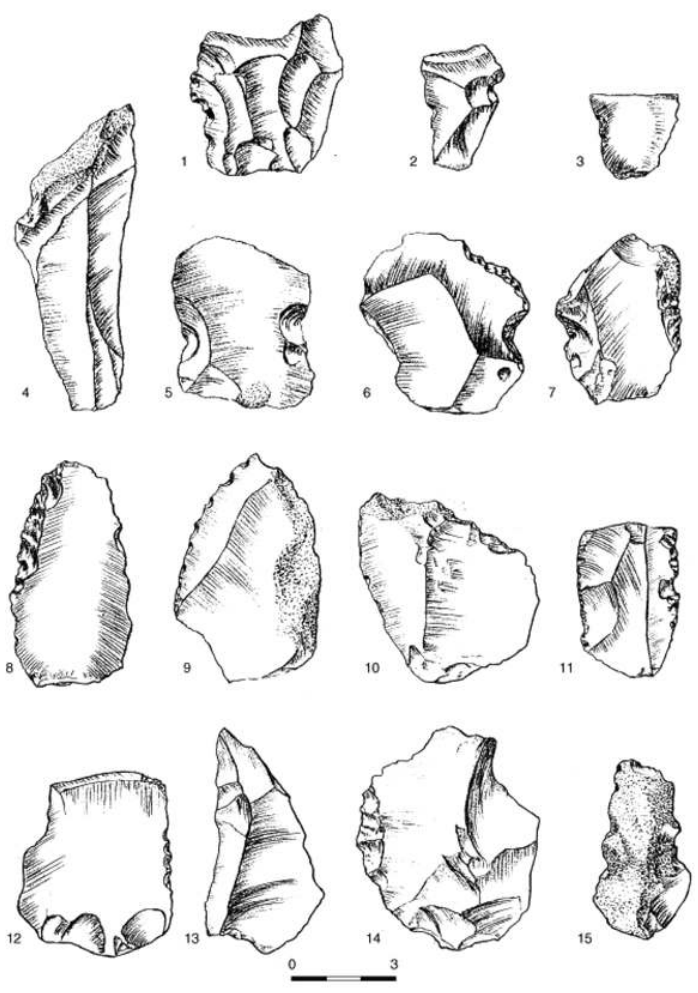

71 Suite à ces différentes considérations, l'attribution de ces industries (couches III et II) au Moustérien typique paraît envisageable. Cependant l'attribution d'une industrie lithique à ce faciès paraît quelque peu réductrice; sa seule caractéristique étant un relatif équilibre entre les principaux groupes d'outils associée à l'absence ou la rareté de certains types d'outils (bifaces, couteaux à dos, racloirs Quina) (Bordes, 1984). Il est peut-être temps de se demander si chaque industrie moustérienne doit être obligatoirement rattachée à l'un des faciès définis par $\mathrm{F}$. Bordes, quitte à masquer une certaine variabilité omniprésente au Paléolithique moyen. Nous rejoignons ici les conclusions de M. Otte selon lesquelles "Il faut sans doute (...) fuir les dogmes et les schémas rigides" (Otte 1996, p. 247)

\section{Le problème de l'attribution d'une série lithique au Moustérien à denticulés}

Au même titre que l'industrie lithique de la couche III du Roc de Marsal, certaines industries provenant des fouilles anciennes ont pu être attribuées au Moustérien à denticulés alors qu'une partie des "denticulés " n'était que le résultat de l'utilisation d'un tranchant brut ou de phénomènes naturels. Plusieurs auteurs ont déjà dénoncé l'attribution abusive de certains ensembles à ce faciès, et $\mathrm{F}$. Bordes le premier. Nous pouvons citer l'exemple du site de Iabroud, couche 9, dans laquelle les denticulés représentent $30 \%$ de l'outillage, mais " peut être que certains d'entre eux sont le produit d'une utilisation " (Bordes 1981, p. 84). Les industries du Pech de l'Azé II offrent aussi un bon exemple du risque d'erreurs que représente la présence de nombreux pseudo- 
outils au sein d'un ensemble lithique. En effet, certaines pièces, issues de la série " usée ", qui présentaient une retouche inverse ou alterne très abrupte, furent classées en 1950 comme indiscutablement intentionnelles du fait " qu'on peut les regrouper en séries morphologiques" (Bordes et Bourgon 1950, p. 383). Cependant, en 1951, après l'étude d'un plus grand nombre de pièces, cette même industrie fut considérée comme le produit d'un phénomène de concassage et les auteurs qualifièrent les pièces retouchées de "pseudo-outils" (Bordes et Bourgon 1951b, p 522, Bordes 1961). La couche 38 de Combe-Grenal a aussi été attribuée au Moustérien à denticulés alors que "dans bien des cas, il est difficile de dire si les encoches sont volontaires ou si elles résultent d'enlèvements dus à l'utilisation du bord brut" (Turq 2000, p. 293). Une étude en cours sur l'industrie lithique de la couche IVb1 de la grotte de l'Hyène montre là encore que la forte proportion d'outils retouchés semble être liée en partie à la présence de nombreux pseudo-outils (Thiébaut en cours).

73 Il apparaît donc délicat d'attribuer certaines séries à ce faciès sur la seule base d'un nombre important d'outils encochés, parfois relativement frustes. Peut-être qu'un réexamen de certaines séries anciennement rattachées au Moustérien à denticulés modifierait en partie la répartition géographique et chronologique de ce faciès.

Il est encore trop tôt pour tenter une caractérisation définitive de ce faciès relativement complexe, mais il semblerait que d'un point de vue typologique, la bonne facture et la prédominance des pièces encochées apparaissent comme deux des caractéristiques décisives pour l'attribution d'une série lithique au Moustérien à denticulés (Thiébaut en cours).

En effet, plus que des caractères quantitatifs trop rigides (racloirs entre $13 \%$ et $3 \%$ ) ou qualitatifs négatifs (racloirs médiocres) qui risquent de soustraire à ce faciès des industries lithiques telles que celles des couches D de Sandougne et de l'abri Brouillaud (Geneste 1985), c'est avant tout la régularité et la diversité des pièces encochées, associées à leur prédominance sur l'ensemble des outils, qui devrait prévaloir lors de l'attribution d'une série à ce faciès.

L'étude en cours de plusieurs industries attribuées au Moustérien à denticulés nous permettra peut-être si ce n'est de le caractériser, du moins de mettre en évidence l'existence d'une variabilité technologique et socio-économique du Moustérien à denticulés.

\section{Remerciements}

Il m'est agréable de remercier V. Mourre, J. Jaubert, L. Slimak et I. Couchoud qui ont bien voulu relire et corriger ces quelques pages. Une mention particulière pour A. Morala, que je tiens à remercier pour son aide lors de la reconnaissance des matières premières de cette industrie. Le renfort inattendu mais bienvenu de Ch. Fortin et $E$. Durand pour le lavage et le marquage des vestiges lithiques mérite un vif remerciement. L'accueil toujours chaleureux de S. Madelaine et $P$. Plichon lors de mes nombreux séjours aux Eyzies mérite mes plus sincères remerciements. Enfin, l'étude de cette industrie n'aurait pu être effectuée sans le consentement du Conservateur et du Conservateur adjoint du Musée National de Préhistoire des Eyzies, J-J. Cleyet-Merle et A. Turq, qu'ils soient donc ici remerciés. Mes derniers remerciements vont enfin aux deux personnes $d u$ comité de lecture qui ont corrigé cet article J. Jaubert et A. Turq. 


\section{BIBLIOGRAPHIE}

ANTIGNAC G. 1998 - Étude du matériel lithique de la couche II du Roc de Marsal, Bordeaux, mémoire de DEA, $113 \mathrm{p}$.

ASSASSI F. 1986 - Recherches sédimentologiques sur la climatochronologie du Würm ancien et de l'interstade Würmien en Périgord, Bordeaux 1, Thèse de doctorat, 203 p.

BARNES A. S. 1938 - Les outils de l'homme tertiaire en Angleterre. Étude critique, L'Anthropologie, t. 48, pp. $217-236$

BARTON M. 1989 - Modèles de variabilité dans les outils du Paléolithique moyen, L'Anthropologie, 93, pp. 307-310.

BENITO DEL REY L. et BENITO ALVAREZ J. M. 1998 - Métodos y materias instrumentales en Préhistoria (La edad de la piedra tallada màs antigua); tome II tecnologia typologia, Salamanca.

BERNARD-GEUELLE S. 1995 - La grotte de Prélétang (Vercors) : étude d'une industrie moustérienne dans le contexte alpin et périalpin, Université de Provence, mémoire de DEA, 114 p.

BEYRIES S. 1987 - Variabilité de l'industrie lithique au Moustérien : approche fonctionnelle sur quelques gisements français, Oxford (UK), B.A.R., 204 p.

BINFORD L. R. 1966 - A preliminary analysis of functional variability in the Moustérian of Levallois faciès, American Anthropologist, 68, pp. 238-295.

BOËDA E. 1993 - Le débitage discoïde et le débitage Levallois récurrent centripète, Bulletin de la Société Préhistorique Française, 90, 6, pp. 392-403.

BORDES F. 1953 - Essai de classification des industries « moustériennes », Bulletin de la Société Préhistorique Française, 50, 7-8, pp. 457-466.

BORDES F. 1961 - Typologie du Paléolithique ancien et moyen, Cahiers du Quaternaire n 1, Ed. du CNRS, 2 t., (1981), 112 p.

BORDES F. 1962-63 - Le Moustérien à denticulés, Archéoloski Vestnik, XIII-XIV, pp. 43-49.

BORDES F. 1970 - Réflexions sur l'outil au Paléolithique, Bulletin de la Société Préhistorique Française, t. 67, fasc. 7, pp. 199-202.

BORDES F. 1981 - Vingt-cinq ans après : le complexe moustérien revisité, Bulletin de la Société Préhistorique Française, t. 78, fasc. 3, pp. 77-87.

BORDES F. 1984 - Leçons sur le Paléolithique : 2. Le Paléolithique en Europe, Paris (FR), CNRS, 459 p.

BORDES F. et BOURGON M. 1950 - Le gisement du Pech de l'Azé-Nord, prise de date et observations préliminaires, Bulletin de la Société Préhistorique Française, 47, pp. 381-383.

BORDES F. et BOURGON M. 1951a - Le complexe moustérien : Moustériens, Levalloisien et Tayacien, L'Anthropologie, t. 55, pp. 1-23.

BORDES F. et BOURGON M. 1951b - Le gisement de Pech de l'Azé-Nord. Campagne 1950-1951. Les couches inférieures à Rhinocéros Mercki, Bulletin de la Société Préhistorique Française, 48, pp. 521-538.

BORDES F. et LAFILLE J. 1962 - Paléontologie humaine : découverte d'un enfant néandertalien dans le gisement du Roc de Marsal, commune de Campagne du Bugue (Dordogne), Compte Rendu de l'Académie des Sciences, Paris, 254, pp. 714-715. 
BOUCHEREAU J. - 1967. Le Roc de Marsal, Bulletin du Spéléologue de Périgueux, 22, pp. 5-7.

BOUCHUD J. 1975 - Étude de la faune du Roc de Marsal, Multigaphié.

BOURGON M. 1957 - Les industries moustériennes et pré-moustériennes du Périgord, Paris, Masson, $143 \mathrm{p}$.

BREUIL H. et LANTIER R. 1951 - Les hommes de la pierre ancienne (Paléolithique et Mésolithique), Paris, $334 \mathrm{p}$.

CHAVAILLON N. 1985 - L'Atérien du Fourn el Hartani au Sahara nord occidental (République algérienne), Bulletin de la Société Préhistorique Française, 82, pp. 307-337.

COUCHOUD I. 2001 - Processus géologiques de formation du site moustérien du Roc de Marsal, Université de Bordeaux 1, DEA, 76 p.

COUCHOUD I. à paraître - Processus géologique de formation du site moustérien du Roc de Marsal, Paléo.

DAVID F., GIRARD M., HARDY M., LHOMME V., ROBLIN-JOUVE A. et THIÉBAUT, C. 2002 - Arcy-surCure: Grotte du Bison, rapport 2002.

DEFLEUR A. 1987 - Les sépultures moustériennes, Université de Provence Aix-Marseille I, Thèse de Doctorat, $305 \mathrm{p}$.

DEFLEUR A. 1993 - Les sépultures moustériennes, Paris, CNRS, 325 p.

DEMARS P.-Y. 1980 - Les matières premières siliceuses utilisées au Paléolithique supérieur dans le bassin de Brive, Bordeaux, Université de Bordeaux I, Thèse de Doctorat, 173 p.

DIBBLE H. 1984 - Interpreting typological variation of Middle Paleolithic scrapers: function, style or sequence of reduction?, Journal of Field Archaeology, 11, pp. 431-436.

FARIZY C., DAVID F. et JAUBERT J. (Dir), 1994 - Hommes et bisons du Paléolithique moyen à Mauran (Haute-garonne), CNRS éd., XXXème suppl.à Gallia Préhistoire, 267 p.

FLENNIKENN J.-J. et HAGGARTY J. 1979 - Trampling as an agency in the formation of edge damage: an experiment in lithic technology, Northwest Anthropol. Research Notes, 13, pp. 208-214.

FOURNIER R-A. 1973 - Les outils sur galets du site Mindelien de Terra-Amata, Marseille, Université de Provence, Thèse de Doctorat, 221p.

GENESTE J-M. 1985 - Analyse lithique d'industries moustériennes du Périgord : une approche technologique du comportement des groupes humains au Paléolithique moyen, Bordeaux, Université Bordeaux I, Thèse de Doctorat, $567 \mathrm{p}$.

GIRARD C. 1978 - Les industries moustériennes de la grotte de l'Hyène à Arcy-sur-Cure (Yonne), XIe supplément à Gallia Préhistoire, 225 p.

INIZAN M-L., REDURON M., ROCHE H. et TIXIER J. 1995 - Technologie de la pierre taillée, Meudon (FR), Centre de Recherches et d'Etudes préhistoriques / CNRS, 199 p.

JAUBERT J., LORBLANCHET M., LAVILLE H., SLOTT-MOLLER R., TURQ A. et BRUGAL J.-P. 1990 - Les chasseurs d'aurochs de La Borde - un site du Paléolithique moyen (Livernon, Lot), Paris, MSH, Documents d'Archéologie Française n² 27, 157 p.

KARNAY G., AUJOULAT N., KONIK S., MAUROUX B., PLUCHERY E. et TURQ A. 1999 - Notice explicative de la carte géologique de France (1/50 000), feuille le Bugue (807), Orléans, 86 p.

KNUDSON R. 1979 - Inference and imposition in the lithic analysis, In: Lithic use-wear analysis. ed. B. Hayden. Academic Press. N.Y pp. 269-281. 
KONIK S. 1999 - Les dépôts de pente du Périgord: distribution et mise en place; origines des matériaux et étapes de l'évolution des versants, Paris 1 , Thèse de Doctorat, 461p. p.

LAFILLE M. 1961 - Le gisement dit « Roc de marsal », Bulletin de la Société Préhistorique Française, 58, fasc. 11-12, pp. 712-713.

LAFILLE M. n. d. - Cahiers de fouilles.

LEGOUX P. 1965 - Détermination de l'âge de l'enfant néandertalien du Roc de Marsal, Revue française d'odontostomatologie, 10, pp. 1-22.

MADRE-DUPOUY M. 1988 - L'enfant du Roc de Marsal (Dordogne, France). Étude analytique et comparative, Institut de Paléontologie Humaine, Thèse de doctorat du Muséum National d'Histoire Naturelle, 396 p.

MALLOUF R-J. 1982 - An analysis of plow-damaged chert artifacts: the brooken Creek Cache, (41HI86), Hill County, Texas, Journal of Field Archaeology, vol. 9, n 1, pp. 81-98.

MANSUR-FRANCHOMME M-E. 1986 - Microscopie du matériel lithique préhistorique : traces d'utilisation, altérations naturelles, accidentelles et technologiques.Exemple de Patagonie, Paris (FR), CNRS, $186 \mathrm{p}$.

Mc BREARTY S., BISHOP L., PLUMMER T., DEWAR R. et CONARD N., 1998 - Tools underfoot: human trampling as an agent of lithic artifact edge modification, American Antiquity, 63, 1, pp. 108-129.

MELLARS P. 1969 - The Chronology of Mousterian industries in the Perigord region of South-West France, Proceeding of the Prehistoric Society, 35, pp. 134- 171.

MORALA A. 1980 - Observations sur le Périgordien, l'Aurignacien et leurs matières premières lithiques en Haut-Agenais, Toulouse, Toulouse, mémoire de l'École des Hautes Études en Sciences Sociales, $191 \mathrm{p}$.

MORTILLET G. 1872 - Classification de l'âge de la pierre, Matériaux pour l'histoire primitive et naturelle de l'Homme, 2ème série, tome 3, pp. 464-465.

MOURRE V. 1994 - Les industries en quartz au Paléolithique moyen. Approche technologique de séries du Sud-Ouest de la France, Paris-X, Mémoire de Maîtrise, 111 p.

MOURRE V. 2003 - Discoïde ou pas Discoïde ? Réflexions sur la pertinence des critères techniques définissant le débitage Discoïde, in : Discoid lithic technology - Advances and implications, Peresani, M., Ed. , Oxford, B.A.R. International Series 1120, pp. 1-18.

NEWCOMER M-H. 1976 - Spontaneous Retouch, Second International Symposium on Flint. Maastricht, 1976. Nederlandse Geologisce Verenigin, Staringin; 3, pp. 62-64.

NIELSEN A-E. 1991 - Trampling the archaeological record: an experimental study, American Antiquity, 56, pp. 483-503.

OTTE M. 1996 - Le sens des faciès au Moustérien, in : La vie préhistorique, Société préhistorique française, Ed. , Dijon, Faton, pp. 244-247.

OWENW.E. 1938 - The Kombewa Culture, Kenya Colony, Man, 38, pp. 203-205.

PEI W. C. 1936 - Le rôle des phénomènes naturels dans l'éclatement et le façonnement des roches dures utilisées par l'Homme préhistorique, Faculté des sciences de Paris, Thèse de doctorat, 78 p.

PEYRONY D. 1930 - Le Moustier, ses gisements, son industrie, ses couches géologiques, Revue Anthropologique, 40, pp. 3-76 et pp. 155-176. 
PLOUX S. 1983 - Étude de débitages expérimentaux : la marque du tailleur, In : Préhistoire et technologie, cahier $n^{\circ} 2$ (U.R.A 28 du C.N.R.S), pp. 109-179.

PRADEL L. 1956 - Les gisements moustériens de Fontmaure, Congrès Préhist. France, XVe session, Poitiers-Angoulême. pp. 87-91.

PROST D.-Ch. 1989 - Enlèvements accidentels, enlèvements d'utilisation et de retouche sur les outils de pierre taillée, Université de Paris X, Thèse de doctorat, 552 p.

PROST D.-Ch. 1993 - Nouveaux termes pour une description microscopique des retouches et autres enlèvements, Bulletin de la Société Préhistorique Française, 90, 3, pp. 190-195.

ROLLAND N. 1990 - Variabilité du Paléolithique moyen : nouveaux aspects, Colloque International de Nemours, 9-11 mai 1988. Mémoires du Musée de Préhistoire. D’Ile-de-France, 3, pp. 69-76.

SERONIE-VIVIEN M-R. et Seronie-Vivien M. 1959 - Étude géologique de l'anticlinal de SaintCyprien (Dordogne), in : 84ème Congrès des Sociétés Savantes, pp. 571-578.

SLIMAK L. 1997-1998 - La variabilité des débitages discoïdes au Paléolithique Moyen, PAM, t. 7-8, pp. 75-88.

SLIMAK L. 2003 - Les Débitages Discoïdes moustériens : évaluation d'un concept technologique, in : Discoid lithic technology - Advances and implications, Peresani, M., Ed., Oxford, B.A.R. International Series 1120, pp. 33-65.

THIÉBAUT C. 2000 - Analyse technologique et économique de la couche III du Roc de Marsal, Aix-enProvence, Université de Provence, Mémoire de Maîtrise, 152 p.

THIÉBAUT C. 2001 - Caractérisation des encoches et des denticulés au Paléolithique moyen : Application d'une nouvelle méthode d'étude aux pièces encochées de Mauran (Haute-Garonne), Aix-en-Provence, Université de Provence, Mémoire de D.E.A, 232 p.

THIÉBAUT C. en cours - Variabilité technologique et socio-économique du Moustérien à denticulés, Université de Provence, Thèse de Doctorat.

TIXIER J. 1958-1959 - Les industries lithiques d'Ain Fritissa, Bulletin d'Archéologie Marocaine, III, pp. 107-249.

TIXIER J. 1963 - Typologie de l'Epipaléolithique du Maghreb, Paris (FR), Arts et Métiers graphiques, $212 \mathrm{p}$.

TIXIER J. et TURQ A. 1999 - KOMBEWA et al., Paléo 11, pp. 135-145.

TRINGHAM R., COOPER G., ODELL G., VOYETEK B. et WIHMAN A. 1974 - Experimentation in the formation of edge damage: A new approach to lithic analysis., Journal of Field Archaeology, 1 , pp. 171-196.

TURQ A. 1977 - Première approche sur le Paléolithique moyen du gisement des Ardailloux, commune de Soturac (Lot), Bulletin de la société des études du Lot, t. XCVIII, fasc. 4, pp. 222-242.

TURQ A. 1979 - L'évolution du Moustérien de type Quina au Roc de Marsal et en Périgord : modification de l'équilibre technique et typologique, Toulouse, mémoire de l'école des Hautes Etudes en Sciences Sociales, $181 \mathrm{p}$.

TURQ A. 1988 - Le Moustérien du type Quina du Roc de Marsal à Campagne (Dordogne) : contexte stratigraphique, analyse lithologique et technologique, Documents D’Archéologie Périgourdine, 3, pp. 5-30.

TURQ A. 1989 - Le squelette de l'enfant du Roc de Marsal : les données de la fouille, Paléo, 1, pp. 47-54. 
TURQ A. 1992 - Le Paléolithique Inférieur et Moyen entre la vallée de la Dordogne et du Lot, Université de Bordeaux, Thèse de doctorat, $780 \mathrm{p}$.

TURQ A. 2000 - Paléolithique inférieur et moyen entre Dordogne et Lot, Paléo, supplément $\mathrm{N}^{\circ} 2$, $456 \mathrm{p}$.

TURQ A., ANTIGNAC G. et ROUSSEL P. 1999 - Les silicifications coniaciennes du Sarladais et du Gourdonnais : inventaire et implications archéologiques, Paléo 11, pp. 145-160.

VAN CAMPO M., BOUCHUD J. et PIVETEAU J. 1962 - Flore accompagnant le squelette d'enfant moustérien découvert au Roc de Marsal, commune du Bugue, (Dordogne) et première étude de la faune du gisement, note, Comptes rendus de l'Académie des Sciences, 254, pp. 897-899.

VAUFREY R.. 1955 - Préhistoire de l'Afrique, tome premier : Maghreb, Paris, Masson, 460 p.

VERJUX Ch. 1988 - Les denticulés moustériens, in : L’Homme de Néandertal, vol. 4 : La Technique, Liège, ERAUL, pp. 197-204.

\section{NOTES}

1. Caractérisé par un indice de racloirs en essentiel compris entre 13 et 3 (Bordes 1953).

2. Une synthèse de ces divers travaux est proposée au sein de notre mémoire de DEA (Thiébaut 2001).

3. Le repositionnement de la couche III de J. Lafille sur la coupe A a été effectué en collaboration avec I. Couchoud. Néanmoins il devra être confronté aux données de terrain qui seront acquises lors des prochaines recherches.

4. En plus des pièces représentées dans le mémoire de DEA de G. Antignac, nous avons pu observer brièvement l'industrie de la couche II du Roc de Marsal lors de notre année de maitrise.

\section{RÉSUMÉS}

L'industrie de la couche III du Roc de Marsal a été attribuée au Moustérien à denticulés. Un réexamen de l'outillage nous a permis de mettre en évidence la présence de pseudo-outils encochés au sein de cet assemblage. Cette révision a eu pour conséquence une nette diminution de la proportion du groupe des denticulés. Au-delà du problème de l'attribution de certaines industries altérées par des agents naturels ou des actions accidentelles au Moustérien à denticulés, nous nous interrogeons sur la pertinence des caractéristiques uniquement typologiques de ce faciès et, par delà, sur le rattachement systématique d'une industrie à l'un des faciès décrits par F. Bordes.

The industry of the layer III from the Roc de Marsal site was attributed to the Denticulate Mousterian. A renewed study of the tool group allowed us to show the presence of notched pseudo-tools among this industry. This revision induced a drastic decrease of Bordes' group IV ratio. In addition the problem of the Denticulate Mousterian diagnosis in the case of industries altered to natural agents or accidental actions, we question whether the exclusively typological 
characteristics of this facies are pertinent and the systematic identification of industries as one of the Bordes' facies is necessary.

INDEX

Mots-clés : Roc de Marsal, Moustérien à denticulés, pseudo-outils

Keywords : Roc de Marsal, Denticulate Mousterian, pseudo-tools

\section{AUTEUR}

\section{CÉLINE THIEBAUT}

UMR 6636, Économies Sociétés et Environnements Préhistoriques, MMSH, Université de Provence, 5, rue du Château de l'horloge, B.P. 647 F-13094 Aix-en-Provence, cedex 2. 